\title{
A Fully Associative, Nonisothermal, Nonlinear Kinematic, Unified Viscoplastic Model for Titanium Alloys
}

S.M. Arnold

Lewis Research Center

Cleveland, Ohio

A.F. Saleeb

University of Akron

Akron, Ohio

M.G. Castelli

NYMA, Inc.

Engineering Services Division

Brook Park, Ohio

Prepared for the

Second Symposium on Thermomechanical Fatigue Behavior of Materials sponsored by the American Society for Testing and Materials Phoenix, Arizona, November 13-18, 1994

National Aeronautics and

Space Administration 
Trade names or manufacturers' names are used in this report for identification only. This usage does not constitute an official endorsement, either expressed or implied, by the National Aeronautics and Space Administration. 


\title{
A Fully Associative, Nonisothermal, Nonlinear Kinematic, Unified Viscoplastic Model For Titanium Alloys
}

\author{
S. M. Arnold \\ National Aeronautics and Space Administration \\ Lewis Research Center \\ Cleveland OH, 44135 \\ A. F. Saleeb \\ Department Civil Engineering \\ University of Akron \\ Akron, OH 44325 \\ M.G. Castelli \\ NYMA, Inc. \\ NASA Lewis Research Center Group \\ BrookPark, Ohio 44142
}

\begin{abstract}
Specific forms for both the Gibb's and complementary dissipation potentials are chosen such that a complete (i.e., fully associative) potential based multiaxial, nonisothermal unified viscoplastic model is obtained. This model possess one tensorial internal state variable (that is, associated with dislocation substructure) and an evolutionary law that has nonlinear kinematic hardening and both thermal and strain induced recovery mechanisms. A unique aspect of the present model is the inclusion of nonlinear hardening through the use of a compliance operator, derived from the Gibb's potential, in the evolution law for the back stress. This nonlinear tensorial operator is significant in that it allows both the flow and evolutionary laws to be fully associative (and therefore easily integrated), greatly influences the multiaxial response under non-proportional loading paths, and in the case of nonisothermal histories, introduces an instantaneous thermal softening mechanism proportional to the rate of change in temperature. In addition to this nonlinear
\end{abstract}


compliance operator, a new consistent, potential preserving, internal strain unloading criterion has been introduced to prevent abnormalities in the predicted stress-strain curves, which are present with nonlinear hardening formulations, during unloading and reversed loading of the external variables. The specific model proposed is characterized for a representative titanium alloy commonly used as the matrix material in $\mathrm{SiC}$ fiber reinforced composites, i.e., TIMETAL 21S. Verification of the proposed model is shown using "specialized" non-standard isothermal and thermomechanical deformation tests.

Keywords: viscoplasticity, nonlinear hardening, TIMETAL 21S, nonisothermal, deformation, multiaxial, correlations, predictions

\title{
1 Nomenclature
}

\section{Invariants}

$\Omega$

$\Phi$

$F$

G

$J_{2}$

$I_{2}$

$H_{1}$

\begin{abstract}
complementary dissipation potential
Gibb's complementary potential

Bingham-Prager threshold function normalized second invariant function second invariant of effective deviatoric stress tensor second invariant of internal deviatoric stress tensor invariant material function
\end{abstract}

\section{Stresses}

$\sigma_{i j}$
$S_{i j}$
$\Sigma_{i j}$
$\alpha_{\gamma}$
$\alpha_{i j}$
$a_{i j}$
$\kappa, \kappa_{o}$
$Y(T)$
Cauchy stress tensor
deviatoric stress tensor
effective deviatoric stress tensor
internal state variables (stress-like)
internal (or back) stress tensor
deviatoric internal stress tensor
drag stress and reference drag stress
yield stress

\section{Strains \\ $e_{i j}, \epsilon_{i j}^{I}, \epsilon_{i j}^{R}, \epsilon_{i j}^{T}$ $\mathcal{A}_{\xi}$ \\ $\mathcal{A}_{i j}$}

total, inelastic, reversable, and thermal strain tensors, respectively conjugate internal state variables (displacement-like)

internal strain tensor 
Material Parameters

$\theta_{\xi}(T), \theta_{r s}(T)$

$C_{i j k l}(T), E_{i j k l}(T), M_{i j}(T)$

$B_{0}(T), B_{1}, R_{\alpha}$

$\mu(T)$

$\beta(T)$

$n, p, q$

$g(G), f(F)$

$\bar{E}\left(\sigma_{i j}, T\right), \bar{H}\left(\alpha_{\gamma}, T\right), z(T)$

$\eta, \eta_{\tan }, \hat{E}$

Miscellaneous

$T, T_{0}$

$Q_{i j k l},(T), L_{i j k l}(T)$

$\delta_{i j}$

\langle\rangle

$\mathrm{Hv}[\mathrm{]}$

$(\cdot)$ dynamic thermal recovery operator

elastic compliance, stiffness and thermal expansion tensor, respectively hardening and thermal recovery material parameters

material parameter associated with the viscosity of the material denotes the extent of strain induced recovery

material exponents

material functions

material functions

mean coefficient of thermal expansion(CTE), instanteous CTE, Young's modulus, respectively

current and reference temperature, respectively

internal compliance and internal stiffness operators, respectively Kronecker delta function

Macauley bracket

Heaviside unit function

time derivative (or rate) notation

\section{Introduction}

A number of Titanium Matrix Composite (TMC) systems are currently being researched and evaluated for high temperature air frame and propulsion system applications. As a result, numerous computational methodologies for predicting both deformation and life for this class of materials are under development. An integral part of these methodologies is an accurate and computationally efficient constitutive model for the metallic matrix constituent. Furthermore, because of the proposed elevated operation temperatures for which these systems are designed, the required constitutive models must account for both time-dependent and time-independent deformations. To accomplish this we will employ a recently developed complete potential based framework [1] utilizing internal state variables which was put forth for the derivation of reversible and irreversible constitutive equations. This framework, and consequently the resulting constitutive model, is termed complete because the existence of the total (integrated) form of the Gibb's complementary free energy and complementary dissipation potentials are assumed a priori. The specific forms selected here for both the Gibb's and complementary dissipation potentials result in a fully associative, multiaxial, nonisothermal, unified viscoplastic model with nonlinear kinematic hardening. Thus this model constitutes one of many models in the GVIPS (Generalized VIscoplasticity with Potential Structure) class of inelastic constitutive equations which can be constructed using the generalized framework of Arnold and Saleeb [1].

The particular unified GVIPS model of interest in this study possesses one tensorial 
internal state variable (i.e., the back or internal stress) that is associated with dislocation substructure and an evolutionary law that has nonlinear kinematic hardening and both thermal and strain induced recovery mechanisms. A unique aspect of the present model is the inclusion of nonlinear hardening through the use of a compliance operator (derived from the Gibb's potential) in the evolution law for the back stress. This nonlinear tensorial operator is significant in that it allows both the flow and evolutionary laws to be fully associative (and therefore easily integrated) [2], greatly influences the multiaxial response under non-proportional loading paths [1],[3],[4], and in the case of nonisothermal histories, introduces an instantaneous thermal softening mechanism proportional to the rate of change in temperature. In addition to this nonlinear compliance operator, the new [5] consistent, potential preserving, internal unloading criterion has been utilized to prevent abnormalities in the predicted stress-strain curves, which are present with nonlinear hardening formulations, during unloading and reversed loading.

The primary objective of the present study is to specify material functions and characterize the associated material parameters for the nonisothermal extension of the previously proposed kinematic, isothermal GVIPS model [5] for TIMETAL 21S ${ }^{1}$, an advanced titanium-based matrix commonly used in TMCs. Although both long and short term behavior is important, capturing the short term (or transient) behavior and rate sensitivity of the material is of primary importance given that the applications of interest are primarily those involving material processing and structural issues in propulsion systems.

The paper begins by briefly summarizing the extension of the complete potential structure to the nonisothermal domain, followed by a multiaxial statement of the newly proposed nonisothermal GVIPS model. A discussion regarding the characterization of the proposed model is then followed by numerous results illustrating the predictive capabilities of the model.

\section{Complete Potential Structure: Nonisothermal}

Here, the basic thermodynamic framework put forth by Amold and Saleeb [1] is extended to the nonisothermal domain. Expressions for the Gibb's thermodynamic and the complementary dissipation potential functions are assumed in terms of a number of state and internal variables characterizing the changing internal structure of the material. For conciseness, the discussion is limited to a case involving i) small deformations (in which the initial state is assumed to be stress free), ii) an initially isotropic material, and iii) the specialized (decoupled) potential framework discussed in [1]. A Cartesian coordinate reference frame and index notation are utilized (repeated Roman subscripts imply summation).

Given the Gibb's potential in the following form

$$
\Phi=\Phi\left(\sigma_{i j}, \alpha_{\gamma}, T, \epsilon_{i j}^{I}\right)
$$

\footnotetext{
${ }^{1}$ TIMETAL $21 \mathrm{~S}$ is a registered trademark of TIMET, Titanium Metals Corporation, Toronto, $\mathrm{OH}$.
} 
and assuming a priori that the inelastic strain is an independent parameter (and not an internal state variable), for example

$$
\Phi=\bar{E}\left(\sigma_{i j}, T\right)-\sigma_{i j} \epsilon_{i j}^{I}+\bar{H}\left(\alpha_{\gamma}, T\right)-\frac{\sigma_{k k}}{3} \eta\left(T-T_{o}\right)
$$

an expression for the total strain rate can be obtained by differentiating, that is,

$$
\dot{e}_{i j}=\frac{d}{d t}\left(\frac{-\partial \Phi}{\partial \sigma_{i j}}\right)=C_{i j r s} \dot{\sigma}_{r s}+\dot{\epsilon}_{i j}^{I}+\left[\frac{-\partial^{2} \bar{E}\left(\sigma_{i j}\right)}{\partial \sigma_{i j} \partial T}+\frac{\delta_{i j}}{3}\left(\eta+\frac{\partial \eta}{\partial T} \Delta T\right)\right] \dot{T}
$$

as well as the rate of change of the conjugate internal variables $\left(A_{\xi}\right)$,

$$
\dot{\mathcal{A}}_{\xi}=\frac{d}{d t}\left(\frac{-\partial \Phi}{\partial \alpha_{\xi}}\right)=Q_{\xi \gamma} \dot{\alpha}_{\gamma}+\theta_{\xi} \dot{T}
$$

where

$$
C_{i j r s}=\frac{-\partial^{2} \Phi}{\partial \sigma_{i j} \partial \sigma_{r s}}=\frac{-\partial^{2} \bar{E}\left(\sigma_{i j}, T\right)}{\partial \sigma_{i j} \partial \sigma_{r s}}
$$

and

$$
Q_{\xi \gamma}=\frac{-\partial^{2} \Phi}{\partial \alpha_{\xi} \partial \alpha_{\gamma}}=\frac{-\partial^{2} \bar{H}\left(\alpha_{\gamma}, T\right)}{\partial \alpha_{\xi} \partial \alpha_{\gamma}}
$$

are the external and internal compliance operators, respectively, and

$$
\theta_{\xi}=\frac{-\partial^{2} \Phi}{\partial \alpha_{\xi} \partial T}=\frac{-\partial^{2} \bar{H}\left(\alpha_{\gamma}, T\right)}{\partial \alpha_{\xi} \partial T}
$$

is the change in the conjugate internal variable $\left(A_{\xi}\right)$ with temperature. Note the three terms in equation (3) may then be identified from left to right as the reversible, irreversible (inelastic), and thermal expansion components of the total strain rate, respectively. Thus,

$$
\dot{e}_{i j}=\dot{\epsilon}_{i j}^{R}+\dot{\epsilon}_{i j}^{I}+\dot{\epsilon}_{i j}^{T}
$$

where

$$
\dot{\epsilon}_{i j}^{R}=C_{i j r s} \dot{\sigma}_{r s}
$$

and

$$
\dot{\epsilon}_{i j}^{T}=M_{i j} \dot{T}
$$

with

$$
M_{i j}=\frac{-\partial^{2} \Phi}{\partial \sigma_{i j} \partial T}=\left[\frac{-\partial^{2} \bar{E}\left(\sigma_{i j}\right)}{\partial \sigma_{i j} \partial T}+\frac{\delta_{i j}}{3} \eta_{\tan }\right]
$$

and

$$
\eta_{\tan }=\eta+\frac{\partial \eta}{\partial T} \Delta T
$$


denoting the instantaneous coefficient of thermal expansion, and $\dot{\epsilon}_{i j}^{I}$ (the inelastic strain rate) is defined separately using the concept of a complementary dissipation potential $\Omega\left(\sigma_{i j}, \alpha_{\gamma}, T\right)$.

Given

$$
\Omega=\Omega\left(\sigma_{i j}, \alpha_{\gamma}, T\right)
$$

and using the Clausius-Duhem inequality; the flow law becomes

$$
\dot{\epsilon}_{i j}^{I}=\frac{\partial \Omega}{\partial \sigma_{i j}}
$$

and the evolutionary laws for the thermodynamic conjugate internal state variables are:

$$
\dot{\mathcal{A}}_{\gamma}=-\frac{\partial \Omega}{\partial \alpha_{\gamma}}
$$

Utilizing equation (4) the internal constitutive rate equations for the internal state variables are obtained,

$$
\dot{\alpha}_{\gamma}=L_{\gamma \xi}\left[\dot{\mathcal{A}}_{\xi}-\theta_{\xi} \dot{T}\right]
$$

where

$$
L_{\gamma \xi}=\left[Q_{\gamma \xi}\right]^{-1}=\left[\frac{-\partial^{2} \Phi}{\partial \alpha_{\xi} \partial \alpha_{\gamma}}\right]^{-1}
$$

Thus, equations (13) and (14) represent the flow and evolutionary laws, for an assumed $\Omega=\Omega\left(\sigma_{i j}, \alpha_{\gamma}, T\right)$, and equation (15) the internal constitutive rate equations, given a Gibb's potential $\Phi$, wherein both potentials are directly linked through the internal state variables $\alpha_{\gamma}$.

\section{Viscoplastic Constitutive Model}

A complete multiaxial statement of a GVIPS model can be derived by using the above framework, given a specific form for both the Gibb's potential, $\Phi$, and the complementary dissipation potential, $\Omega$. Form invariance (objectivity) of these potentials, and material symmetry considerations requires that they depend only on certain invariants of their respective tensorial arguments (i.e., an integrity basis [6] ). In the spirit of von Mises and because of the deviatoric nature of inelastic deformation, only the quadratic invariant will be considered at this time in specifying the dissipation potential. Similarly, only the linear elastic strain energy contribution will be considered in specifying the Gibb's potential, with the internal state groupings being functions of the respective quadratic invariants. Finally, although equation (12) indicates that an unlimited number of internal state variables can be specified, here our attention will be restricted to a GVIPS model with a single independent, evolving, internal state variable. This internal state variable, 
$a_{i j}$, is taken to be a second order symmetric traceless tensor that represents the internal (or back) stress associated with dislocation substructure. Two additional scalar state variables associated with dislocation density are considered. One representing the drag strength $(\kappa)$ is taken to be temperature dependent and yet non-evolving with respect to plastic work; whereas, the other, a yield stress $(Y)$, is uniquely assumed to implicitly evolve with internal stress over a specified temperature range.

Consequently, the Gibb's potential may be written as

$$
\Phi=-\frac{1}{2} C_{r s k l}(T) \sigma_{r s} \sigma_{k l}-\sigma_{i j} \epsilon_{i j}^{I}+H_{1}(G, T)-\frac{\sigma_{k k}}{3} \eta\left(T-T_{o}\right)
$$

and the complementary dissipation potential as

$$
\Omega=\mu(T) \int f(F(T)) d F+R_{\alpha}(T) B_{0}(T) \int g(G) d G
$$

where

$$
\begin{gathered}
F(T)=\left\langle\frac{\sqrt{J_{2}}}{\kappa(T)}-Y(T)\right\rangle \\
Y(T)=\langle 1-\beta(T) \sqrt{G}\rangle \\
G=\frac{I_{2}}{\kappa_{0}^{2}} \\
H_{1}(G)=-B_{0}(T)\left(G+B_{1} G^{p}\right) \\
I_{2}=\frac{3}{2} a_{i j} a_{i j} \\
J_{2}=\frac{3}{2} \Sigma_{i j} \Sigma_{i j} \\
\Sigma_{i j}=S_{i j}-a_{i j} \\
S_{i j}=\sigma_{i j}-\frac{1}{3} \sigma_{k k} \delta_{i j} \\
a_{i j}=\alpha_{i j}-\frac{1}{3} \alpha_{k k} \delta_{i j} .
\end{gathered}
$$

and $\kappa_{0}=\kappa\left(T_{0}\right)$ where $T_{0}$ is the reference temperature.

Note that in the preceding expression for the dissipation potential, the stress dependence, both external and internal, enters through the scalar functions $F$ and $G$ in the form of effective $\left(\Sigma_{i j}\right)$ and internal $\left(a_{i j}\right)$ deviatoric stresses, respectively. Furthermore, the function $F$ acts like a threshold surface, since when $F<0$, no inelastic strain can occur. Clearly, this threshold value is dictated by the magnitude of both the drag strength 
$(\kappa)$ and yield stress $(\mathrm{Y})$. A unique aspect of this model is that the internal variable representing the yield stress is specifically taken as a special scaled function of the back stress and drag strength. Consequently, this allows for 1) the model to possess features of a model with three internal variables yet without any additional computational cost, and 2) the presence of an induced strain recovery term (as opposed to the common 'ad-hoc' introduction of such terms) in the evolution of the back strain (i.e., the associated conjugate variable, $\mathcal{A}_{\gamma}$ ) at lower temperatures. It is important to realize that the product $\kappa Y$ constitutes the radius of the initial threshold surface, thereby dictating from physical arguments, that both $\mathrm{Y}$ and $\kappa$ be always positive valued. Furthermore, given the specified form of $Y$ in equation (20), it is clear that the material parameter ratio $(\beta / \kappa)$ will dictate the limit value (i.e., when $Y=0)$ of the internal stress $\left(\alpha_{i j}\right)$, or the cut-off limit for dynamic recovery.

By selecting the preceding scalar functions, a general yet complete potential-based model, with associated flow and evolutionary laws, can be constructed. The second invariants , $J_{2}$ and $I_{2}$, are also scaled for tension. These invariants could just as easily have been scaled for shear by replacing the coefficient $3 / 2$ with $1 / 2$, and modifying the definition of the magnitude of the inelastic strain rate that follows. Also, it should be stated that the linear forms of $F$ and $Y$ in equations (19) and (20) were chosen in order to allow algebraic manipulation and analytical solution of the resulting expressions (e.g., inversion of the flow law), so as to ease the characterization stage of the model, as discussed in [5].

Taking the appropriate derivatives of both $\Phi$ and $\Omega$ as indicated in equations (3) through (16), one obtains the multiaxial nonisothermal specification particular to the present constitutive model. Here the decomposition of the total strain rate is that of equation (8), where the reversible mechanical and thermal strain rate is given by equations (9) and (10) and the irreversible (or inelastic) component is defined by the following flow law:

$$
\dot{\epsilon}_{i j}^{I}=\frac{3}{2}\left\|\dot{\epsilon}_{i j}^{I}\right\| \frac{\Sigma_{i j}}{\sqrt{J_{2}}} \quad \text { if } \quad F \geq 0
$$

or

$$
\dot{\epsilon}_{i j}^{I}=0 \quad \text { if } \quad F<0
$$

where

$$
\left\|\dot{\epsilon}_{i j}^{I}\right\|=\sqrt{\frac{2}{3} \dot{\epsilon}_{i j}^{I} \dot{\epsilon}_{i j}^{I}}=\frac{\mu(T) f(F(T))}{\kappa(T)}
$$

The internal constitutive rate equation is always given by

$$
\dot{a}_{i j}=L_{i j r s}\left[\dot{\mathcal{A}}_{r s}-\theta_{r s} \dot{T}\right]
$$

while the evolutionary law for the back strain rate is given by : 


$$
\dot{\mathcal{A}}_{k l}=\dot{\epsilon}_{k l}^{I}-\frac{3}{2} \frac{\beta(T)}{\kappa_{o}^{2}} \kappa(T)\left\|\dot{\epsilon}_{i j}^{I}\right\| \frac{a_{k l}}{\sqrt{G}} H v[Y]-\frac{3 R_{\alpha}(T) B_{0}(T) g(G)}{\kappa_{o}^{2}} a_{k l} \quad \text { if } \quad a_{i j} \Sigma_{i j} \geq 0
$$

during internal loading and

$\dot{\mathcal{A}}_{r s}=Q_{r s l m} E_{l m n p}\left(\dot{\epsilon}_{n p}^{I}-\frac{3}{2} \frac{\beta(T)}{\kappa_{o}^{2}} \kappa(T)\left\|\dot{\epsilon}_{i j}^{I}\right\| \frac{a_{n p}}{\sqrt{G}} H v[Y]-\frac{3 R_{\alpha} B_{0} g(G)}{\kappa_{o}^{2}} a_{n p}\right) \quad$ if $\quad a_{i j} \Sigma_{i j}<0$

during internal unloading, as indicated by the inequality $a_{i j} \Sigma_{i j}<0$. The internal stiffness operator is defined as

$$
L_{i j r s}=\left[Q_{i j r s}\right]^{-1}=\frac{\kappa_{o}^{2}}{3 B_{0}(T)\left(1+B_{1} p G^{p-1}\right)}\left(I_{i j r s}-\frac{3 B_{1}(p-1) G^{p-2}}{\kappa_{o}^{2}\left(1+B_{1} p G^{p-1}(6 p-5)\right)} a_{r s} a_{i j}\right)
$$

with $I_{i j r s}=\delta_{i r} \delta_{j s}$; whereas, the dynamic thermal recovery operator, is defined as:

$$
\theta_{r s}=\frac{\partial B_{0}(T)}{\partial T}\left(1+B_{1} p G^{p-1}\right) \frac{3}{\kappa_{o}^{2}} a_{r s}
$$

Equation (33) constitutes a new consistent, potential preserving, internal unloading criterion that prevents the classical abnormalities [7] in the cyclic response associated with nonlinear hardening formulations, upon unloading and reversed loading of the external variables as described in [5]. Justification for this criterion stems from the work of Orowan [8] and others, and has indicated that upon stress reversals dislocations are remobilized and consequently rapid rearrangement of these dislocations is possible within the wake of the previous load path. To describe this rapid motion of dislocation remobilization (readjustment of internal stress) during material unloading we introduce distinct regions within the state space in which the rate of the conjugate internal state variable (internal strain) changes discontinuously. Currently, until more exploratory tests can be performed to fully describe the affected regions of the state space, the internal (back) strain rate during internal unloading is taken to be proportional to the back strain rate during loading, through the product of the external stiffness and internal compliance operators, see eq. (33). Thus, implying that the internal stress and strain during internal unloading are related by the external stiffness tensor. Furthermore, an examination of the evolution of the conjugate of the internal stress (i.e., the internal strain, $\mathcal{A}_{i j}$ ), clearly shows that equation (32) possesses, as typically assumed in the literature ( cf. [9] through [13]), competitive mechanisms consisting of a hardening term (which accounts for strengthening mechanisms) and two state recovery terms (which account for softening mechanisms). The first state recovery term evolves with inelasticity; it is strain induced, and is commonly called dynamic recovery; whereas, the second term, interchangeably called static or thermal recovery, evolves with time and is thermally induced. The added 


\begin{tabular}{|c|c|c|}
\hline Constants & Units & TIMETAL 21S \\
\hline Viscoplastic & & \\
$\kappa$ & $\mathrm{MPa}$ & 5.86 \\
$\mu$ & $\mathrm{MPa} / \mathrm{sec}$ & $5.52 \times 10^{-9}$ \\
$\mathrm{n}$ & - & 3.3 \\
$B_{0}$ & $\mathrm{MPa}$ & $5.86 \times 10^{-4}$ \\
$B_{1}$ & - & 0.05 \\
$\mathrm{p}$ & - & 1.8 \\
$\mathrm{q}$ & - & 1.35 \\
$R_{\alpha}$ & $1 / \mathrm{sec}$ & $0.1 \times 10^{-5}$ \\
$\beta$ & - & 0.01 \\
Elastic & & 80,671 \\
$\mathrm{E}$ & $\mathrm{MPa}$ & 0.365 \\
$\nu$ & - & \\
\hline
\end{tabular}

Table 1: Isothermal material parameters for TIMETAL $21 \mathrm{~S}$ at $650^{\circ} \mathrm{C}$

flexibility provided by the dynamic recovery term is particularly advantageous at low homologous temperatures where thermal recovery is inactive.

Finally, the above expressions are further specialized by assuming the nonlinearity of inelasticity and the thermal recovery process to be represented by power law functions, that is:

$$
\begin{aligned}
& f(F)=F^{n} \\
& g(G)=G^{q}
\end{aligned}
$$

\subsection{Characterization}

Given the previously characterized isothermal GVIPS model [5] with the nine material parameters corresponding to the reference temperature, $\mathrm{T}_{0}$, of $650^{\circ} \mathrm{C}$, the extension of the model to the nonisothermal regime becomes straightforward with the primary task being associated with the determination of the number and functional form of the required temperature dependent parameters. Table 1 lists both the elastic ( $\mathrm{E}$ and $v$ ) and inelastic material parameters utilized in the isothermal model; that is the three parameters associated with the flow law (i.e., $\kappa, \mu$, and $\mathrm{n}$ ), the three with the nonlinear hardening operator, (i.e., $B_{0}, B_{1}$, and p), two with the internal thermal recovery term (i.e., $R_{\alpha}$ and $q$ ), and the one parameter with the strain induced (dynamic) recovery term (i.e., $\beta$ ). Considering the physical and mathematical significance (see eqs. (28)-(34)) of the above material parameters one could easily argue that only five of the nine inelastic parameters, in addition to the elastic stiffness and coefficient of thermal expansion, need be functions of temperature. Clearly, since the magnitude of $\kappa$ defines the onset of inelastic (or irreversible) behavior, i.e., through the threshold surface F, one would expect 


\section{Parameters}

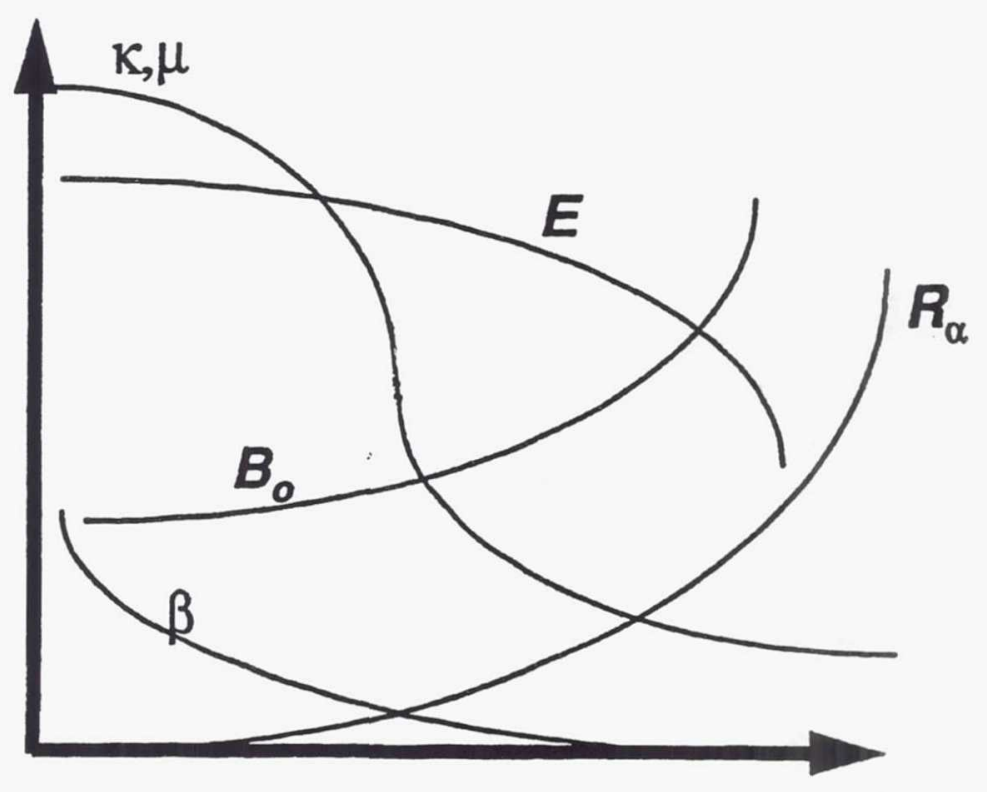

\section{Temperature}

Figure 1: Schematic illustrating the temperature dependence of the elastic and inelastic material parameters

its temperature dependence to be a decreasing function as temperature increases, as depicted schematically in Fig. 1. A similar temperature dependence would be expected for the coefficient $\mu$ as it is related to the viscosity of the material, which is known to significantly decrease as the temperature is increased. Two key assumptions that simplify the nonisothermal characterization process considerably, are first, that the internal $\left(L_{i j k l}\right)$ and external $\left(E_{i j k l}\right)$ stiffness operators have a similar temperature dependence (decreasing with increasing temperature, see Fig. 1). Consequently $B_{0}$ is taken initially to have the inverse temperature dependence of the Young's modulus $E(T)$. Second, the exponent $\mathrm{n}$ in the flow law is taken to be temperature independent, thus minimizing the non-unique determination of the remaining material parameters $\mu$ and $\kappa$, and ensuring such theoretical niceties as convexity of the flow surface at all temperatures. Finally, as $\beta$ scales the dynamic recovery term (which is active at low homologous temperatures and inactive at high homologous temperatures) and $R_{\alpha}$ scales the static (thermal) recovery term (which is inactive at low homologous temperatures and active at high) each parameter must have the temperature dependence illustrated in Fig. 1.

As previously stated, the material of choice for characterizing the current nonisothermal GVIPS formulation is the titanium matrix alloy, TIMETAL 21S. This alloy was selected due to the considerable attention it has received for use in TMCs with application toward advanced airframe structures. TIMETAL $21 \mathrm{~S}$ is a metastable $\beta$-titanium alloy with a nominal composition of Ti-15Mo-3Nb-3Al-0.2Si (wt. \%). The coupon spec- 
imens used in this study were taken from "fiberless" panels. The fiberless panels were fabricated by hot isostatic pressing $0.13 \mathrm{~mm}$ thick TIMETAL $21 \mathrm{~S}$ foils, so as to subject the matrix material to an identical processing history as that seen by the matrix material in the composited form. All specimens were subjected to a pre-test heat treatment, consisting of an 8 hour soak in vacuum at $621^{\circ} \mathrm{C}$, to stabilize the $\beta+\alpha$ microstructure of the TIMETAL 21S. For further material, machining, and experimental details see Castelli et al. [14]. All isothermal and nonisothermal tests addressed are uniaxial experiments, thus implying that the multiaxial material constants are typically generalized from their uniaxial counterparts. This need for generalization is precisely why a consistent multiaxial theory, such as that developed from a potential formulation, is imperative. The available tests for use in characterizing the current nonisothermal extension of the GVIPS model are, five tensile tests and four creep tests (performed at a specified reference stress level of $103 \mathrm{MPa}$ ) spanning the representative domains in temperature. Very few repeats were performed due to the limited amount of available material.

The temperature dependence of the elastic properties were obtained by fitting simple polynomial functions through the experimental data [14]. The resulting continuous functions utilized for the present study are taken to be:

$$
\begin{gathered}
E(T)=114141 .\left[1-1.071 \times 10^{-7} T^{2} \ln (T)\right] \\
\eta_{\tan }(T)=4.539 \times 10^{-6}+3.078 \times 10^{-6} \exp \left(\frac{T}{719.6}\right)
\end{gathered}
$$

where the units of $\mathrm{E}(\mathrm{T})$ are $\mathrm{MPa}$ and the temperature, $\mathrm{T}$, is in degrees Celsius. Note, although the poisson's ratio was shown [14] to have a slight temperature dependence the parameter was taken here, for convenience, to be temperature independent and remain at the constant value given in Table 1.

To obtain the actual temperature dependent functional forms for the five inelastic parameters, $\kappa, \mu, B_{0}, R_{\alpha}$, and $\beta$ the following procedure was followed. The first and most important step was identifying the value of the drag strength as this represents the value of stress below which only elastic (or reversible) behavior is "observed", i.e., no measurable inelastic behavior occurs, and this parameter implicitly influences the determination of all other parameters. The actual numerical value employed is typically non-unique as it is dependent upon the definition of inelasticity employed and thus implicitly dependent upon the sensitivity of the experimental equipment. A typical approach taken to arrive at a value for $\kappa$, is to conduct a sequence of creep or relaxation probing tests to determine the maximum value of stress for which no time dependent behavior $\left(\epsilon^{I}=0\right)$ is observed. Such a detailed experimental program was under taken by Castelli et al. [14] to obtain this time dependent threshold. In the process, however, it was discovered that TIMETAL $21 \mathrm{~S}$ exhibits both a time and temperature dependent reversible (linear viscoelastic) and irreversible (viscoplastic) domain ${ }^{2}$. Consequently, an alternative experimental procedure

\footnotetext{
${ }^{2}$ Inclusion of this significant time dependent reversible domain along with the irreversible (viscoplastic) domain will be reserved for a later study.
} 


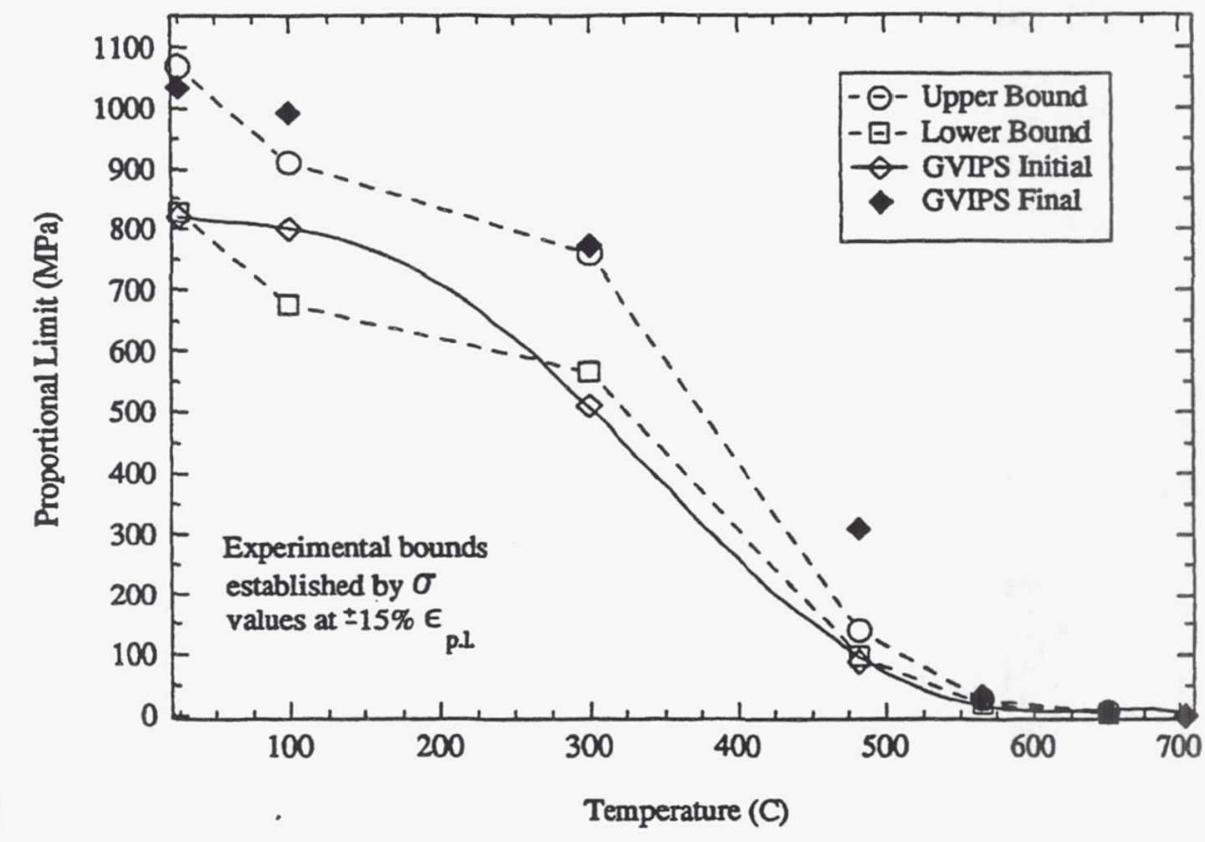

Figure 2: The upper and lower bounds on the experimentally measured drag stress and the initial and final correlation values employed.

was conducted to identify the temperature dependent, irreversible domain $(F>0)$ wherein the viscoplastic inelastic strain component defined in eq. (28) is active. The procedure explained in [14], consists essentially in conducting a number of extremely slow ( $\dot{e}=$ $0.5 \times 10^{-6}$ ), strain controlled tensile tests at various temperatures and looking for the stress value, above which, the stress strain curve deviates from proportionality. In this way the material itself provides the definition of inelasticity, independent of any a priori assumption regarding the accumulation of inelastic strain over a specified time. Given the results of these tests (relative to a conservative and non-conservative definition, i.e., the stress at $\pm 15 \%$ of the proportional limit strain) the drag stress is seen to possesses a sigmoidal like functional dependence on temperature, see Fig. 2. The initial temperature dependent function for $\kappa$ is taken to be:

$$
\kappa(T)=-0.55+\frac{121.2}{\left(1+\exp \left(\frac{(T-333 .)}{71.2}\right)\right.}
$$

and is shown, relative to the experimentally obtained values in Fig. 2.

Given the drag stress as a function of temperature, and the initial temperature dependence of $B_{0}$ taken to be;

$$
B_{0}(T)=B_{0}\left(T_{0}\right) \frac{1.41}{\left[1-1.071 \times 10^{-7} T^{2} \ln (T)\right]}
$$


and taking the initial temperature dependence of $\mu$ to $\mathrm{be}^{3}$

$$
\mu(T)=\frac{\mu\left(T_{0}\right)}{\kappa_{0}} \kappa(T) z(T)
$$

one can then proceed to determine the required $\mu$ (or function $z(\mathrm{~T})$ ) and $\beta$ to simulate the experimental tensile response at the given temperatures. Obtaining these parameters, one would turn to the creep response at the given temperatures to obtain the magnitude of thermal recovery $\left(R_{\alpha}\right)$ required to simulate these histories. Given the assumed temperature independence of the exponent $n$ and the elected use of continuous functions, the effort and compromises required to establish the present set of five temperature dependent inelastic parameters ${ }^{4}$ is minimal in comparison to the effort required to establish the nine inelastic isothermal parameters at the reference temperature of $650^{\circ} \mathrm{C}$. Note that in the above format, $z(\mathrm{~T})$ represents the familiar diffusivity parameter commonly employed in other viscoplastic models in the literature, e.g., see [7],[10] and [11].

This original set of parameters with the single power-law format for rate sensitivity as used here, was found to lead to significant rate sensitivity even at low temperatures. This behavior was not found to be characteristic of this material, however. Therefore, a final recalibration of $\kappa, \mu$ and $\mathrm{B}_{0}$ was undertaken; such that $\kappa$ was increased to approximately its upper bound throughout the temperature range, $\mu$ was increase by at least three orders of magnitude at the lower temperatures and $\mathrm{B}_{0}$ was similarly reduced by a factor of 2 to 6 over the lower temperature regime. The final set of temperature dependent inelastic parameters for the present nonisothermal extension of the GVIPS model are given in Table 2, and also shown in Fig. 2.

The corresponding correlation for the tensile response of TIMETAL 21S (at 25, 300, 482,565 and $650^{\circ} \mathrm{C}$ given a total strain rate of $8.33 \times 10^{-5} / \mathrm{sec}$ ) and short term creep response (at $482,565,650$ and $704^{\circ} \mathrm{C}$ at the reference stress level of $103 \mathrm{MPa}$ ) are shown respectively, in Figs. 3 and 4 . As an aside, note that the way in which one chooses to interpolate between the discrete temperatures given in Table 2 is somewhat arbitrary. For example, one might select the simplest approach of a piecewise linear interpolation, particularly in the case of a significant number of known discrete points, or elect to use continuous functions whenever possible (as done in this study). Note, applying either approach will achieve similar predictive behavior, however, the use of continuous functions allows one to analytically determine the temperature dependent parameters and slopes (see eq. (35)) at all temperatures. The specific material parameter interpolation functions selected for this study are given in appendix $\mathrm{A}$.

The previously [5] discussed rate dependent tensile response at $650^{\circ} \mathrm{C}$ over three orders of magnitude in total strain rate (i.e., $\dot{e}=8.33 \times 10^{-4}, 8.33 \times 10^{-5}$ and $8.33 \times 10^{-6} / \mathrm{sec}$, compliments of Ashbaugh and Khobaib [15]) , short term creep at three stress levels (i.e.,

\footnotetext{
${ }^{3}$ This form for $\mu$ is assumed as the magnitude of inelastic strain is scaled by the ratio $\frac{\mu}{\kappa}$ and the viscosity has a similar temperature dependence as does $\kappa$.

${ }^{4}$ Which accurately simulated the available tensile and creep responses over the given temperature range.
} 


\begin{tabular}{|c|c|cccccc|}
\hline \multicolumn{2}{|c|}{ TIMETAL 21S } & \multicolumn{7}{c|}{ Temperature, ${ }^{\circ} \mathbf{C}$} \\
\hline Constants & Units & $\mathbf{2 5}$ & $\mathbf{3 0 0}$ & $\mathbf{4 8 2}$ & $\mathbf{5 6 5}$ & $\mathbf{6 5 0}$ & $\mathbf{7 0 4}$ \\
\hline$\kappa$ & $\mathrm{MPa}$ & 1034. & 772. & 310. & 33. & 5.86 & 0.75 \\
$\mu$ & $\mathrm{MPa} / \mathrm{sec}$ & 689. & 138. & $6.89 \times 10^{-3}$ & $5.86 \times 10^{-7}$ & $5.52 \times 10^{-9}$ & $7.10 \times 10^{-12}$ \\
$B_{0}$ & $\mathrm{MPa}$ & $6.89 \times 10^{-5}$ & $1.03 \times 10^{-4}$ & $1.72 \times 10^{-4}$ & $4.86 \times 10^{-4}$ & $5.86 \times 10^{-4}$ & $6.36 \times 10^{-4}$ \\
$R_{\alpha}$ & $1 / \mathrm{sec}$ & 0. & 0. & $1.679 \times 10^{-7}$ & $1.685 \times 10^{-7}$ & $1.0 \times 10^{-6}$ & $6.0 \times 10^{-5}$ \\
$\beta$ & - & 0.001 & 0. & 0. & 0. & 0. & 0.0 \\
\hline
\end{tabular}

Table 2: Nonisothermal material parameters for TIMETAL 21S.

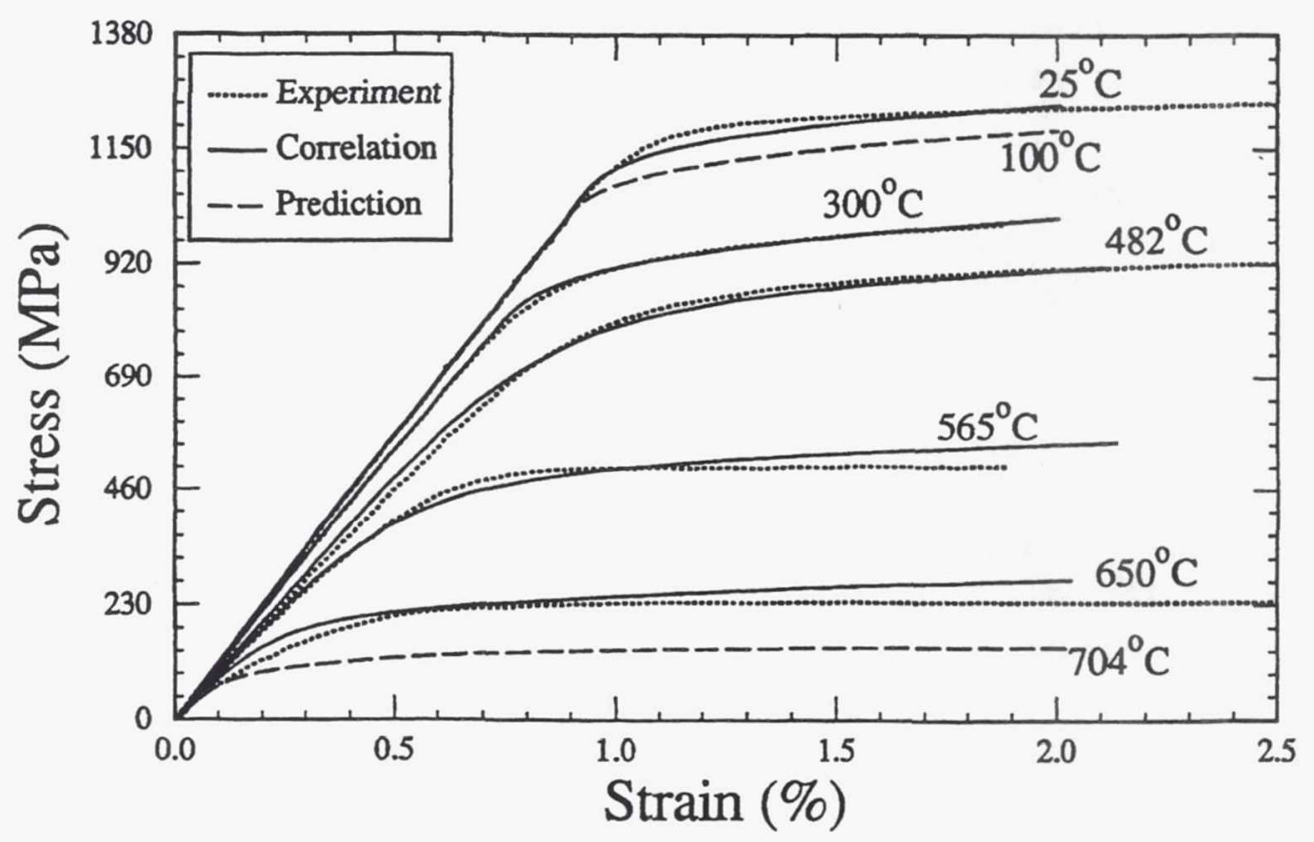

Figure 3: GVIPS correlation with experimental tensile data at 25, 300, 482, 565 and $650^{\circ} \mathrm{C}$, given a total strain rate of $8.33 \times 10^{-5} / \mathrm{sec}$. 


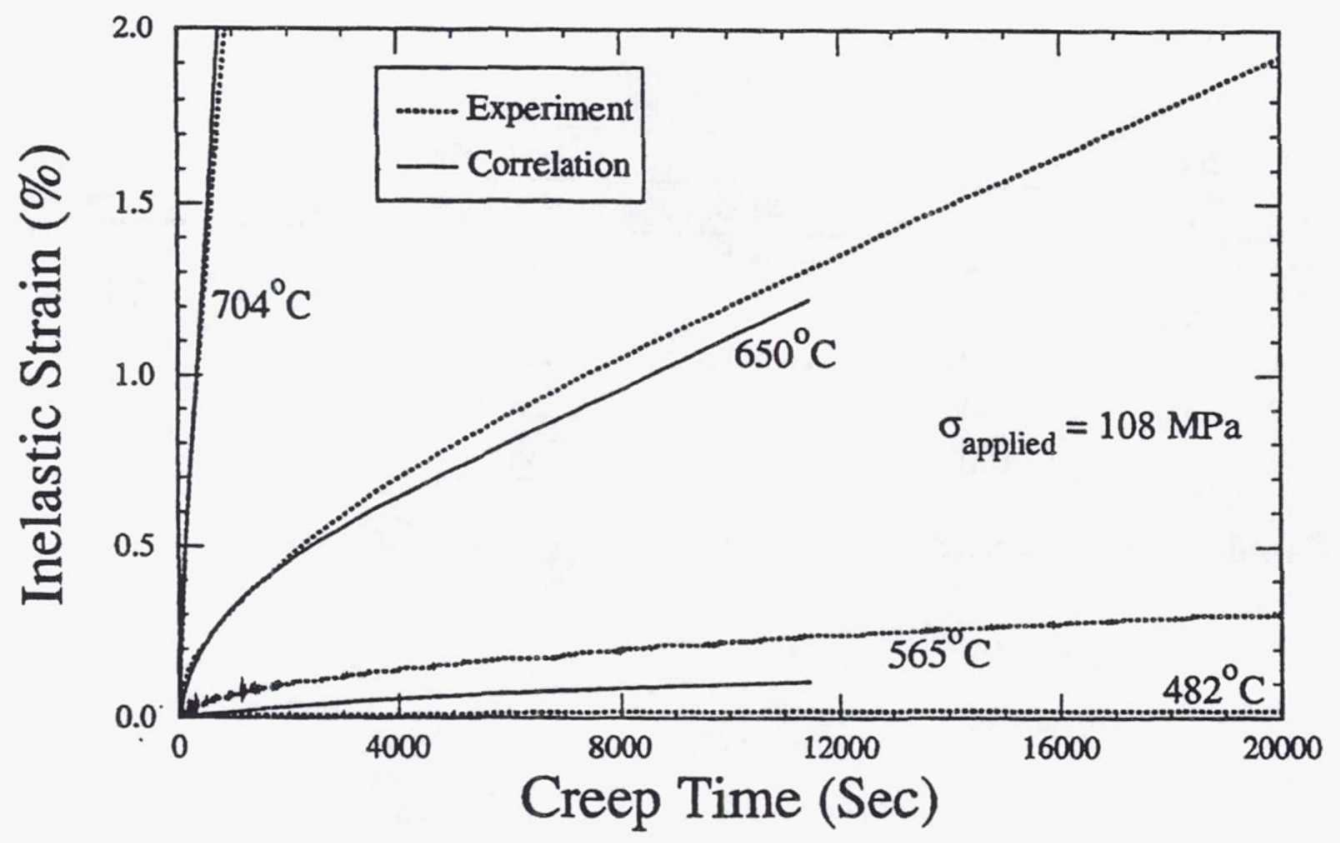

Figure 4: GVIPS correlation with experimental creep data, at the reference stress level of $15 \mathrm{ksi}$ and three temperatures, 565,650 , and $704^{\circ} \mathrm{C}$.

$\sigma=72,110$, and $128 \mathrm{MPa}$ ), and relaxation at 103,238 and $345 \mathrm{MPa}$, are repeated here in Figs. 5, 6 and 7, respectively, for completeness. In particular, figure 5 illustrates the present model's capability to capture the significant rate dependence of this material at $650^{\circ} \mathrm{C}$, whereas figures 6 and 7 demonstrate the accurate stress-level sensitivity of the model under both creep and relaxation conditions. In addition, note the ability of the model, in figure 6, to accurately represent both the primary and secondary creep regions. Clearly, the present model does an excellent job, given the wide variety of loading conditions and temperature range examined. This is particularly true when one considers the relatively small number of temperature dependent and independent material constants required to simulate these histories.

\section{GVIPS Predictions}

The present nonisothermal GVIPS model, characterized to represent the behavior of TIMETAL 21S from 23 to $704^{\circ} \mathrm{C}$, is now exercised and its predictive capability assessed. 


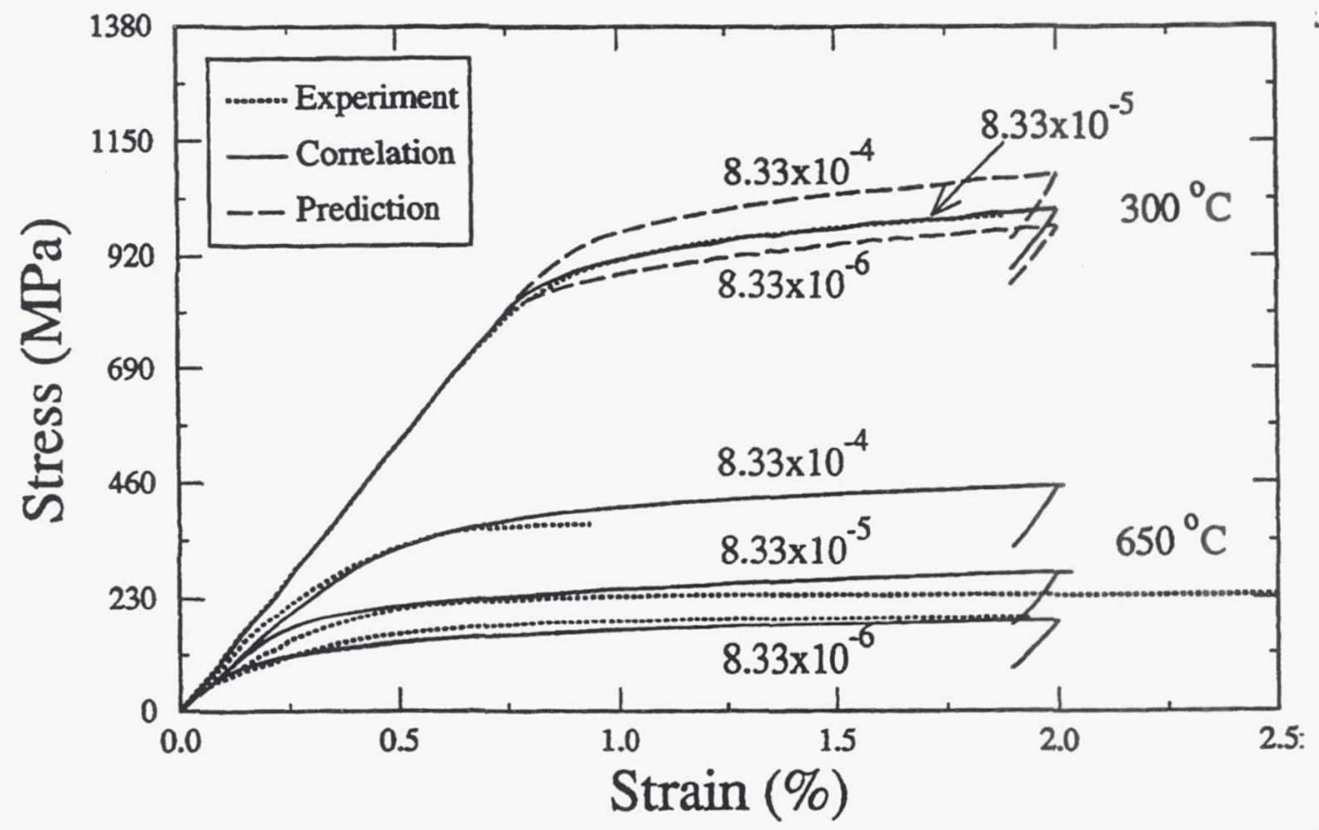

Figure 5: GVIPS correlation and prediction of strain rate sensitivity at 300 and $650^{\circ} \mathrm{C}$; 650 data taken from [13].

\subsection{Relaxation Behavior}

The assessment begins by considering relaxation tests performed at stress levels above and below that used (i.e., $\sigma=238 \mathrm{MPa}$ ) in the characterization of the model at the reference temperature of $650^{\circ} \mathrm{C}$, see Fig. 7. Clearly, the overall agreement with short term behavior is quite good with the initial stress rates being accurately predicted at the higher stress levels and yet diminishing more rapidly than do the actual experimental values at the lower stress levels. Note that the three starting stress levels in Fig. 7 represent stress levels ${ }^{5}$; i) below the apparent yield, ii) within the knee (e.g. 115 to $330 \mathrm{MPa}$ ), and iii) near the ultimate stress level of the material (approximately 385 $\mathrm{MPa}$ ), see Fig. 5. As mentioned earlier, the significant reversible time dependent domain observed in TIMETAL 21S has not been included in the present model; therefore, it is not surprising that the relaxation response at the lower stress levels within the apparent elastic regime are not as accurately represented. Consequently, attention was focused on the relaxation response of TIMETAL $21 \mathrm{~S}$ at 300 and $565^{\circ} \mathrm{C}$ given a considerable prior total strain history of $1.9 \%$. The corresponding stress-strain and stress-time test and model prediction results for these two temperatures are shown in Figs. 8 and 9 respectively. Clearly, both short and mid term behaviors are accurately predicted.

Multistep relaxation tests were performed to document the influence of prior history

\footnotetext{
${ }^{5}$ Given a $5.0 \times 10^{-4}$ total strain rate ramp-up history, see Fig. 5.
} 


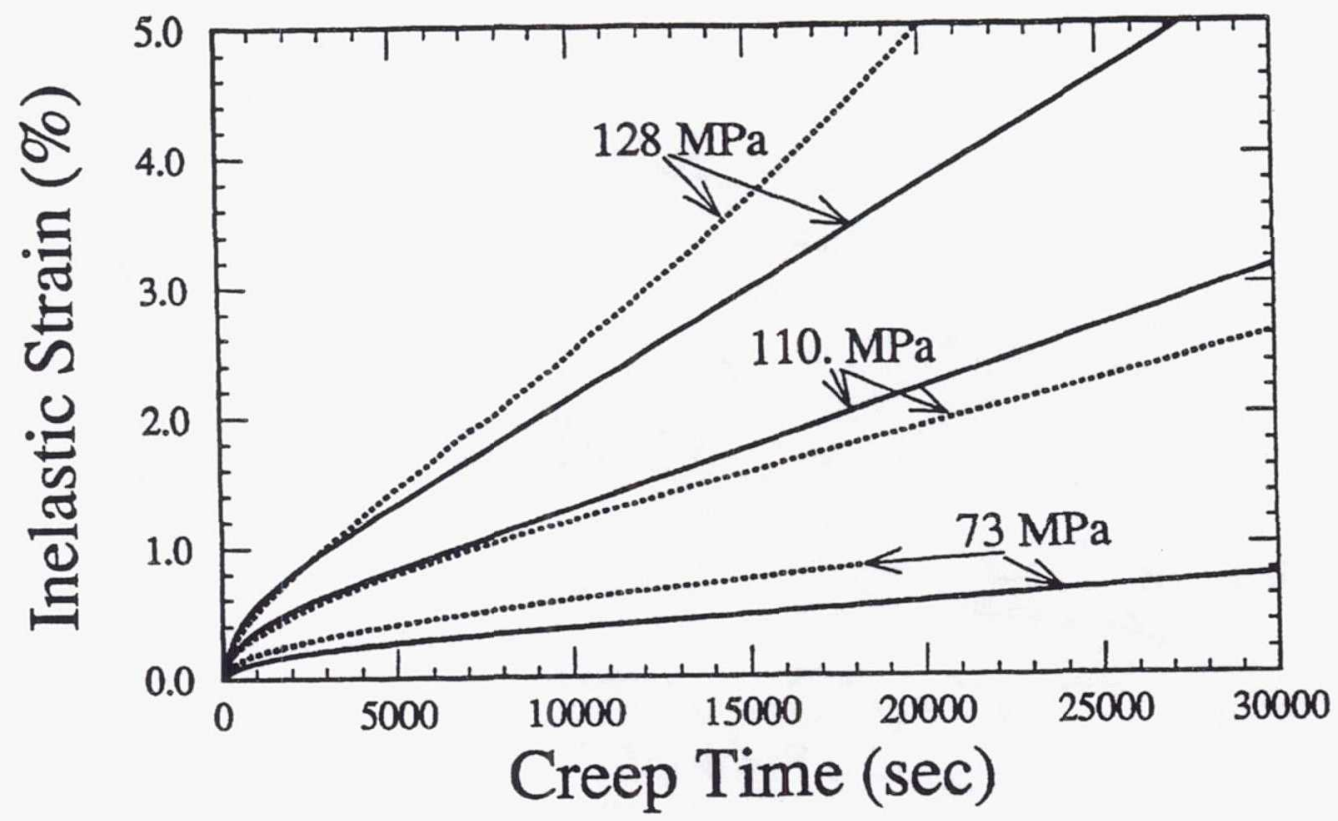

Figure 6: GVIPS correlation (solid lines) of experimental creep data (dotted lines) under various stress levels at the reference temperature, $650^{\circ} \mathrm{C}$.

on the time dependent response of TIMETAL $21 \mathrm{~S}$ and validate the predictive capability of the present model. One such test consisted of a two step load sequence comprised of a $345 \mathrm{MPa}$ stress level, relaxed for 24 hours (i.e., step A) followed by an initial 103 MPa stress level relaxed for approximately one hour (i.e., step B). The experimental stress versus time response and GVIPS simulation for step B are shown in Fig. 10. To illustrate the influence of prior history on the relaxation behavior of TIMETAL $21 \mathrm{~S}$ at $650^{\circ} \mathrm{C}$, the stress-time response for the initial stress level of $103 \mathrm{MPa}$ without prior history (i.e., the virgin history) given in Fig. 7 has also been included in Fig. 10. Clearly, prior history has a significant influence on the subsequent response and GVIPS is fully capable of accurately predicting such influence. Similarly, a three step relaxation load sequence was investigated as shown in the insert of Fig. 11, wherein step 1 began at a stress level of $103 \mathrm{MPa}$, step 2 at $238 \mathrm{MPa}$ and step 3 once again at $103 \mathrm{MPa}$ with each constant total strain hold period lasting approximately 24 hours. The resulting experimental (dotted lines) and GVIPS (solid lines) stress versus time response for each step is given in Fig. 11. Again, it is clearly demonstrated that the presently characterized GVIPS model is fully capable of accurately capturing the influence of multistep load histories. 


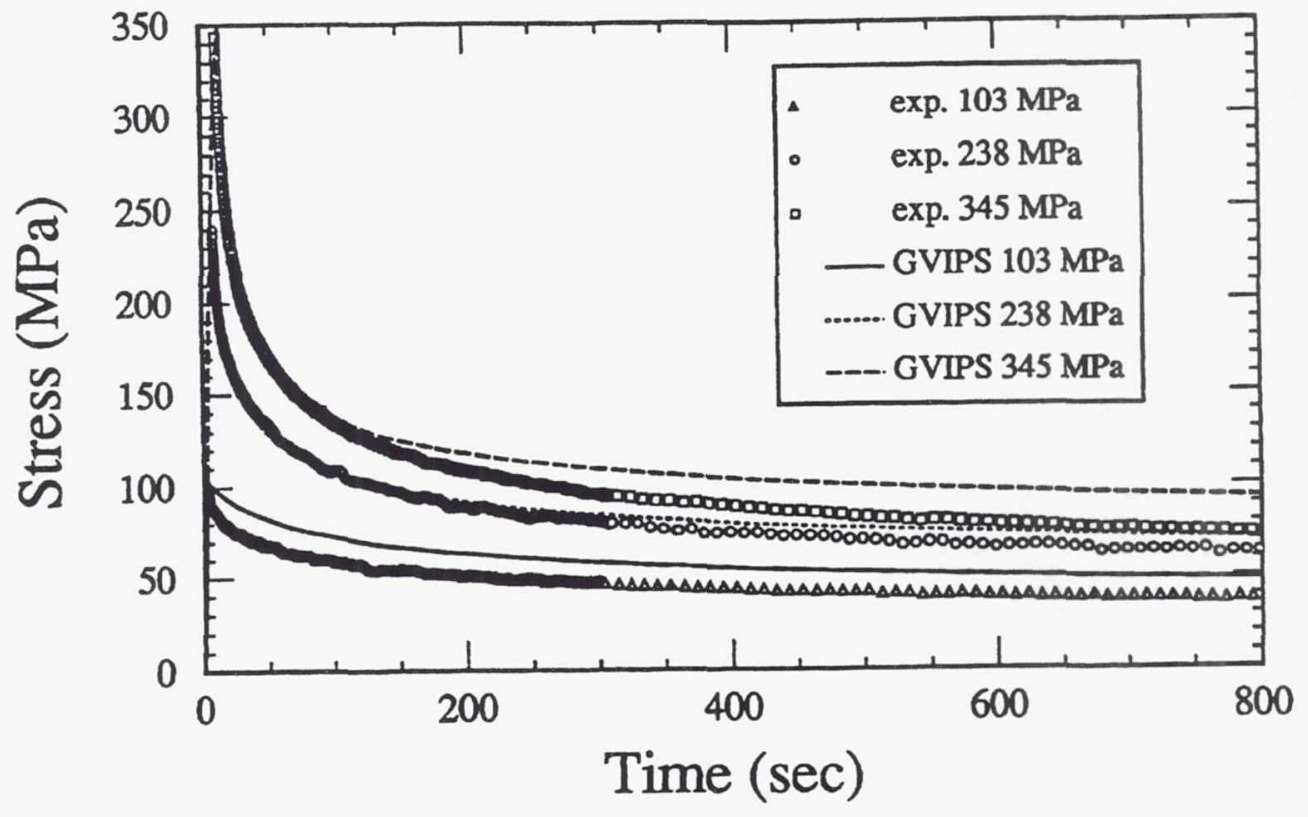

Figure 7: Short term relaxation response of TIMETAL $21 \mathrm{~S}$ at $650^{\circ} \mathrm{C}$ : GVIPS simulation (lines) versus experimental results (symbols). Initial loading total strain rate of $5.0 \mathrm{x}$ $10^{-4} / \mathrm{sec}$. 


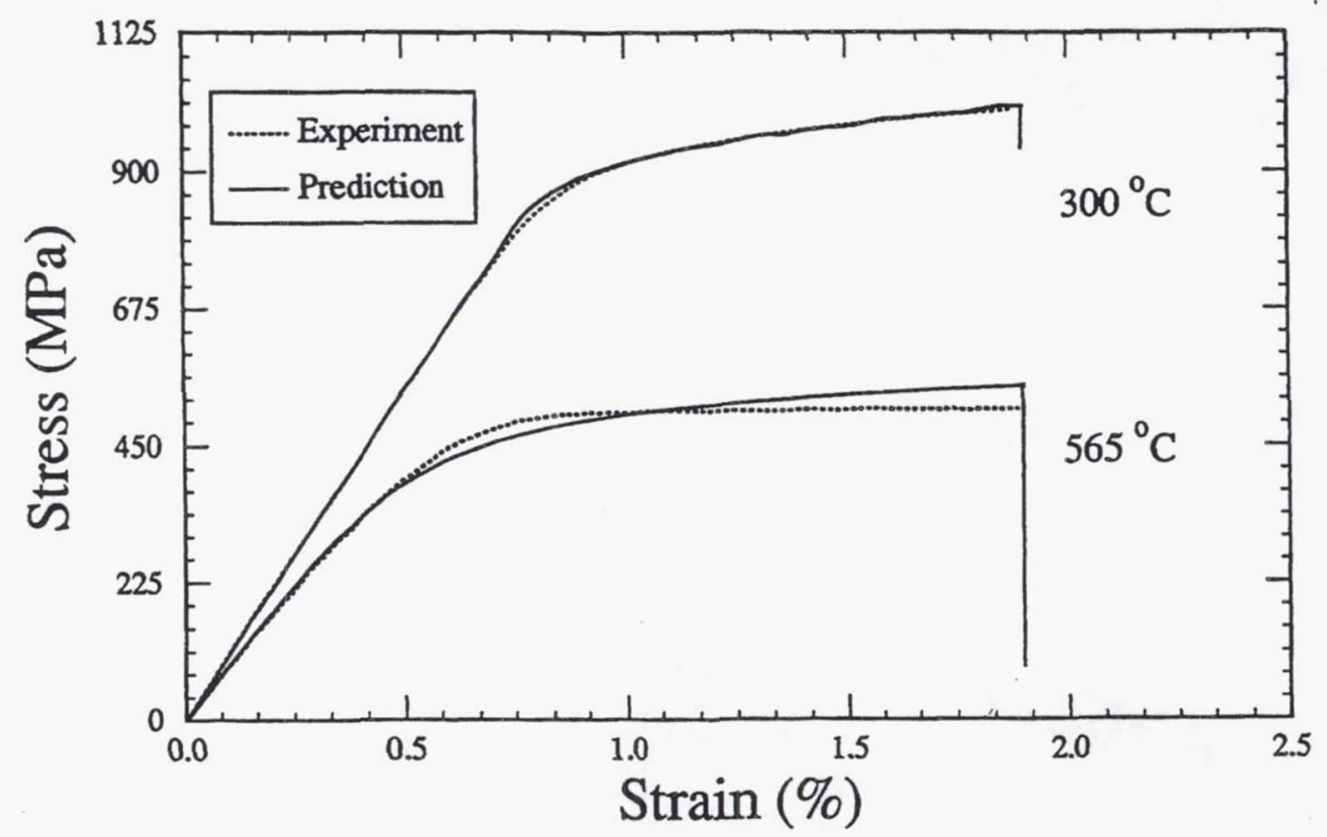

Figure 8: The experimental and GVIPS stress-strain response resulting from relaxation tests at 300 and $565^{\circ} \mathrm{C}$.

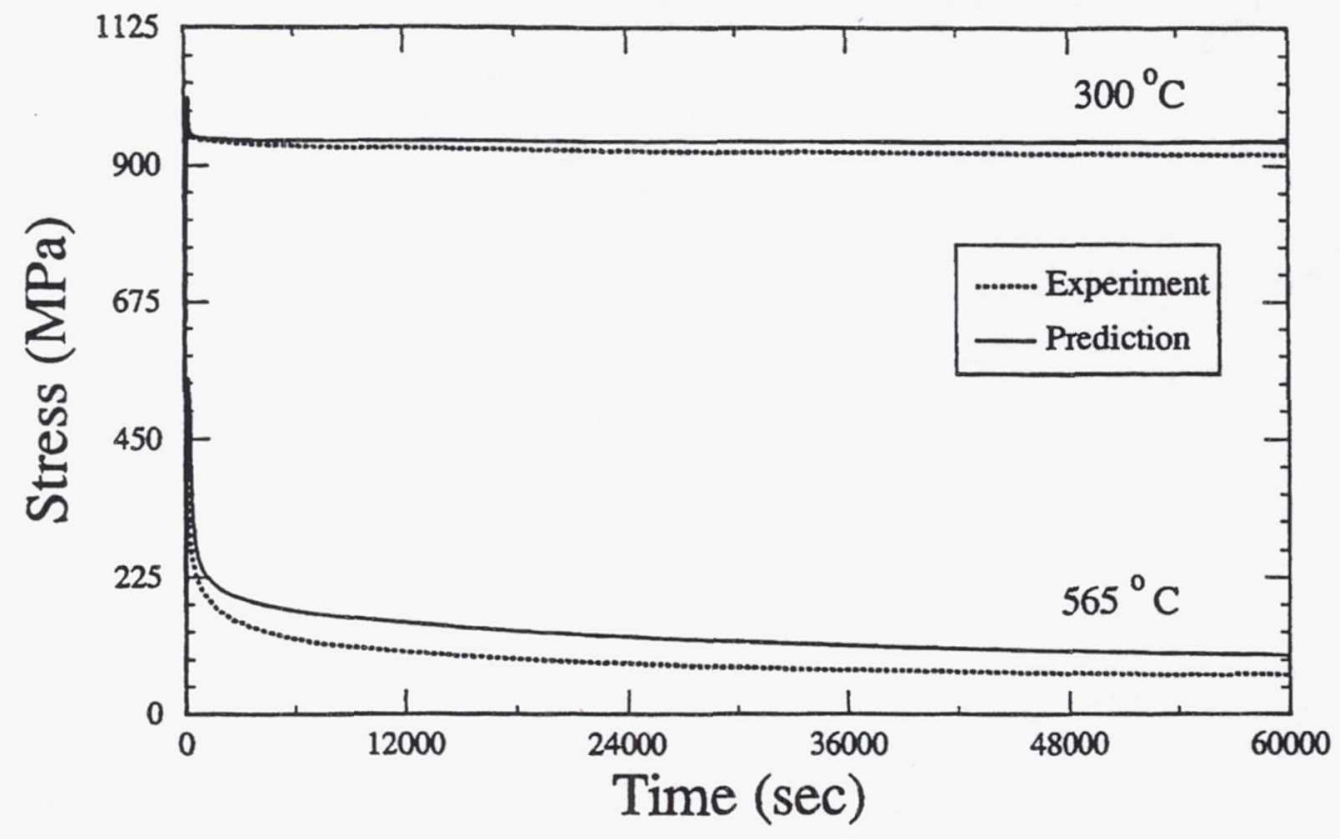

Figure 9: The experimental and GVIPS stress-time response resulting from two relaxation tests at 300 and $565^{\circ} \mathrm{C}$. 


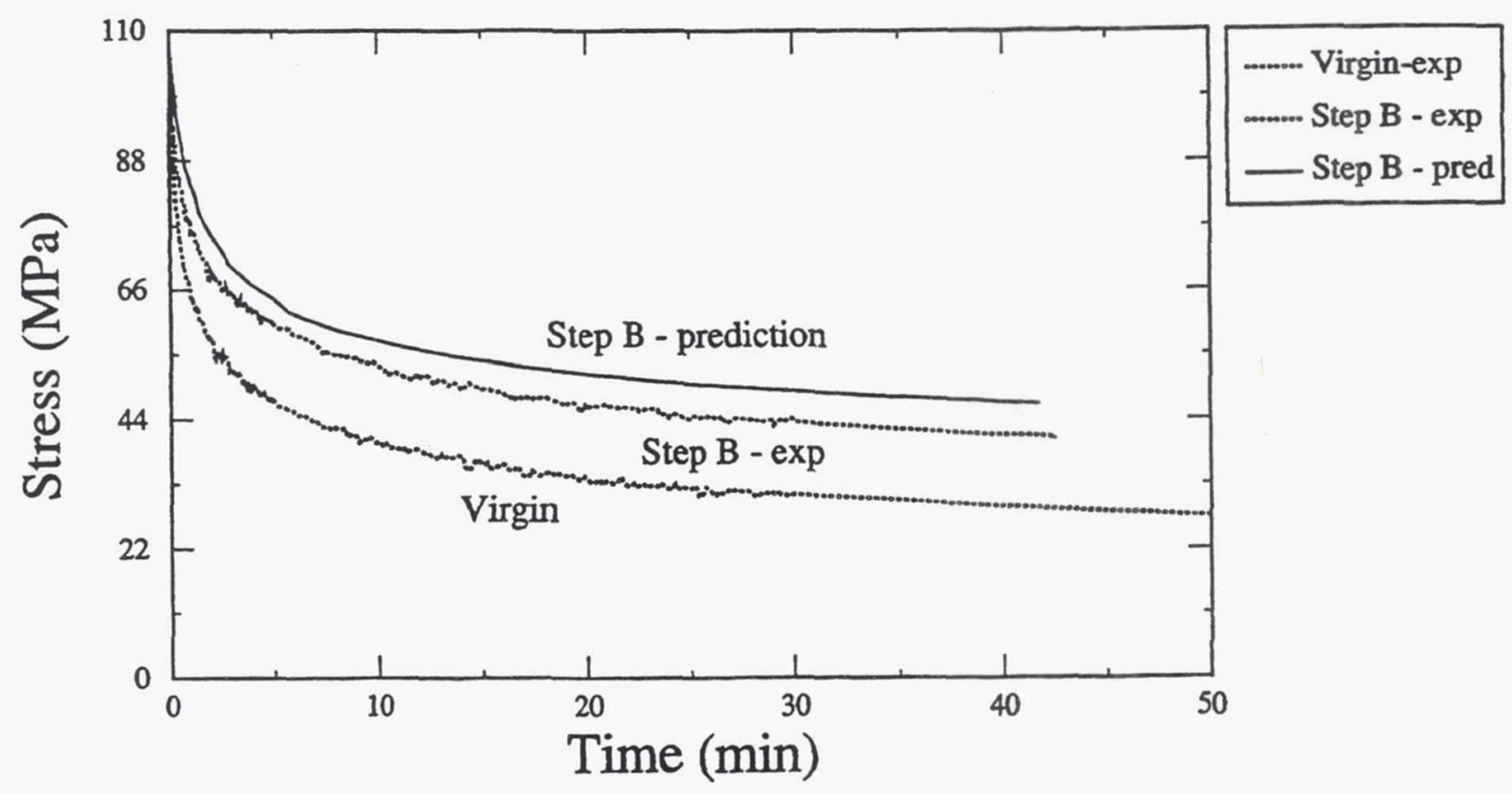

Figure 10: Two step relaxation test at $650^{\circ} \mathrm{C}$; Step B is the relaxation response subsequent to Step A.

\subsection{Rate Sensitivity}

Figure 5 illustrates the rate sensitivity of the present model at $300^{\circ} \mathrm{C}$ and at the reference temperature $650^{\circ} \mathrm{C}$, given a variation in the applied strain rate of three orders of magnitude. The accurate correlation of the rate sensitivity at $650^{\circ} \mathrm{C}$ is evident and, although the rate sensitivity is reduced as the temperature is decreased, the present model is still considered to over-predict the rate sensitivity of TIMETAL 21S at lower temperatures. The difficulties associated with predicting a highly rate sensitive material at elevated temperatures, as compared with a rate insensitive material at lower temperatures are believed to be enhanced because of neglecting the significant reversible, time dependent domain present at elevated temperatures in this material. As stated previously, inclusion of this feature will be the focus of our future research.

\subsection{Cyclic Behavior}

Given a total strain rate of $8.33 \times 10^{-5} / \mathrm{sec}$, the cyclic stress-strain behavior of the present nonisothermal model is demonstrated for three temperatures, i.e., 25,482 , and $650^{\circ} \mathrm{C}$ as illustrated in Fig. 12 [15]. The cyclic response at 650 is considered to be a correlation with experiment whereas those at 482 and $25^{\circ} \mathrm{C}$ are actual predictions. Obviously, the room temperature cyclic behavior agrees quite well with experimental data. Furthermore, 


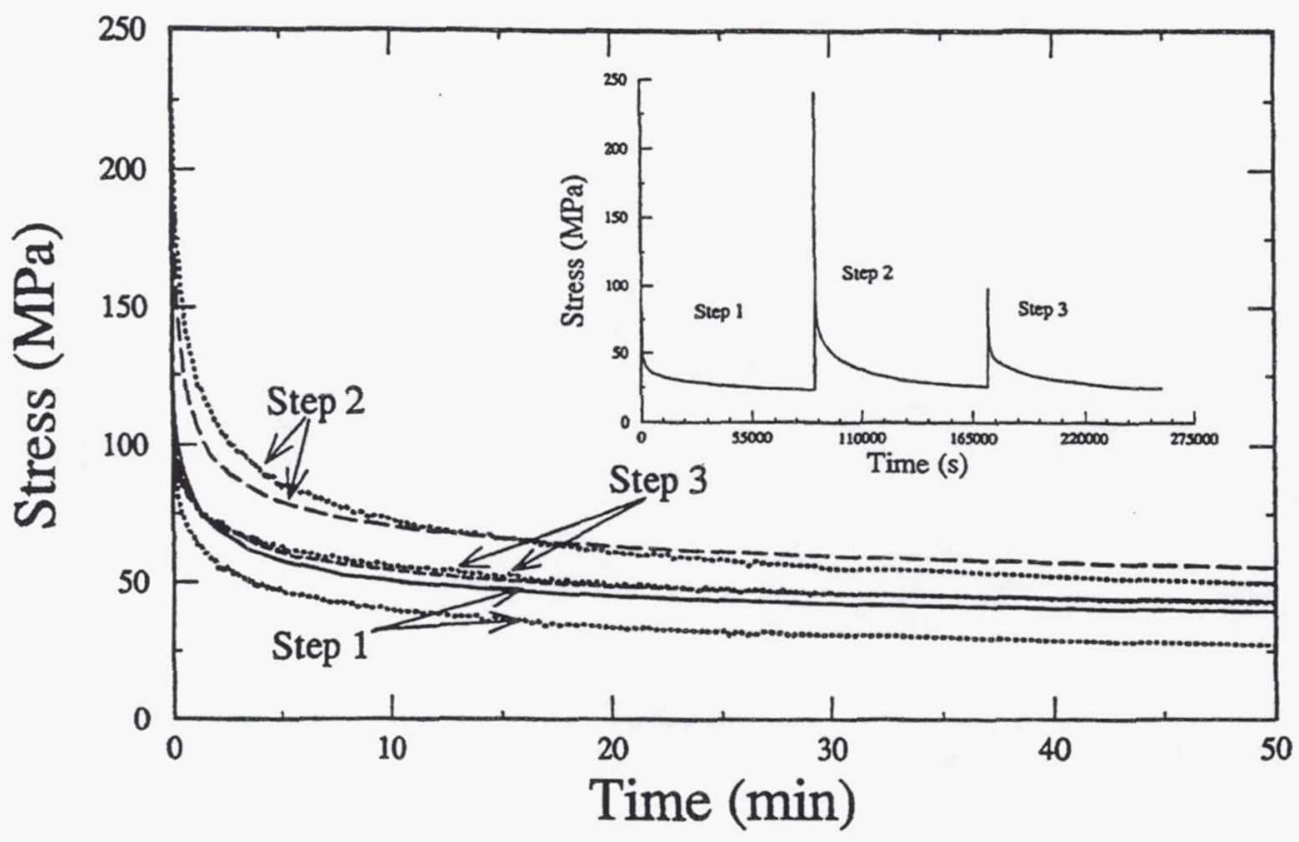

Figure 11: Three step relaxation test at $650^{\circ} \mathrm{C}$ as described in the insert.

these cyclic results illustrate that the consistent, potential preserving, internal unloading criterion prevents, over a wide temperature range, the classical abnormalities in the cyclic stress-strain response associated with nonlinear hardening formulations, even with the exclusion of any isotropic hardening component.

\subsection{Temperature Step Test}

In order to examine the significance of the new dynamic thermal recovery term, resulting from the nonisothermal extension of the GVIPS model, a multiple-step temperature creep test was conducted. This test began with a $103 \mathrm{MPa}$ creep test at $565^{\circ} \mathrm{C}$, followed by a temperature step, under zero load, to $650^{\circ} \mathrm{C}$; whereupon a $103 \mathrm{MPa}$ creep test is once again conducted. This history was then followed by another temperature step, under zero load, to $704^{\circ} \mathrm{C}$ and a subsequent $103 \mathrm{MPa}$ creep test. The resulting experimentally measured creep strain versus creep time response (dotted line) is shown in Fig. 13 along with the GVIPS prediction (solid line). Comparing the model simulation to the experimental results it is apparent that the model under-predicts the material creep response at $565^{\circ} \mathrm{C}$ by approximately $35 \%$. This is not surprising, as the correlation of the $565^{\circ} \mathrm{C}$ creep response was similarly under predicted, see Fig. 6. However, several key qualitative features of the temperature step test are represented with reasonable accuracy, including the re-initiation of primary creep subsequent to the steps, and the overall total strain accumulation. 


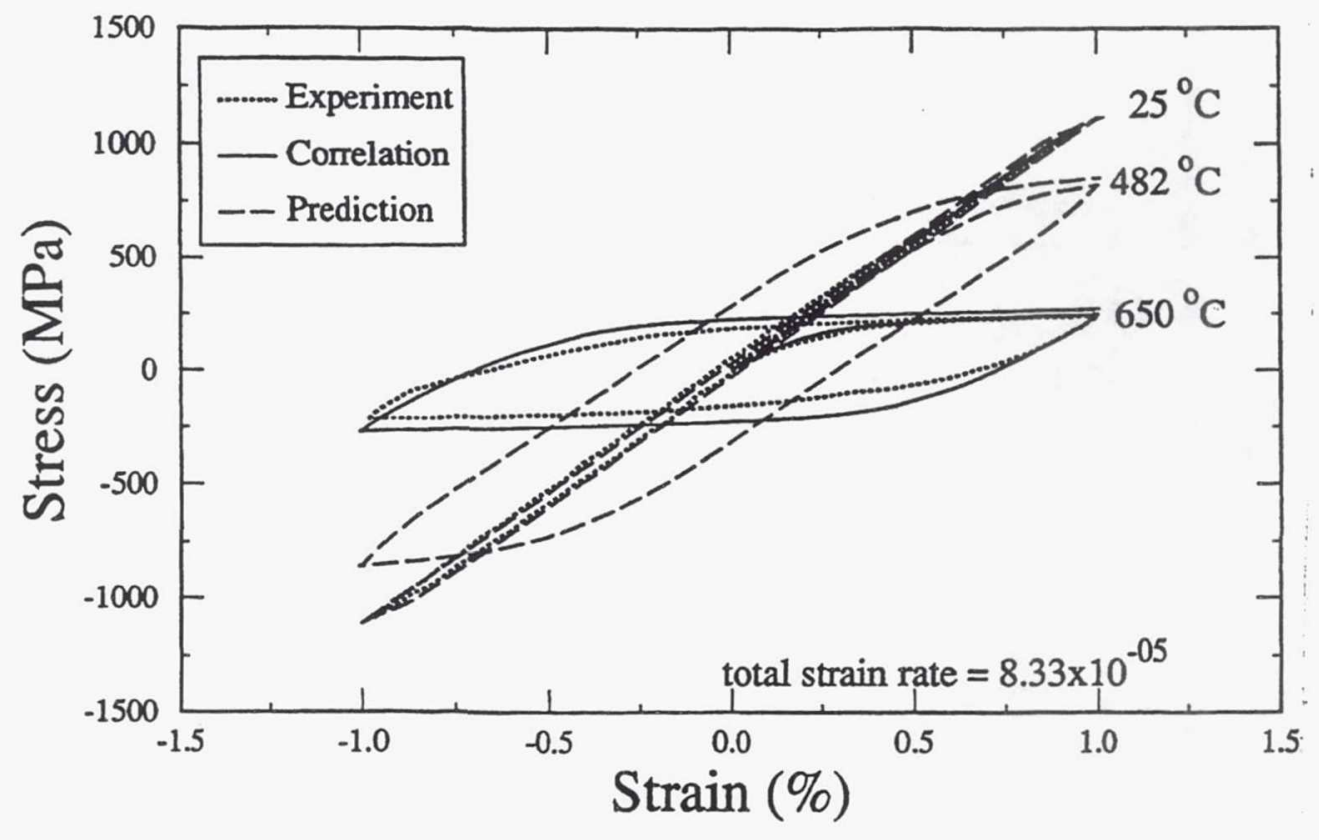

Figure 12: GVIPS simulation of cyclic stress-strain behavior at 25,482 and $650^{\circ} \mathrm{C}$ [13].

One interesting aspect of this step test as discussed in Castelli et al. [14] is the increased primary creep rate, over that of the virgin sample, subsequent to the jump in temperature to $650^{\circ} \mathrm{C}$, thus suggesting the need for a dynamic thermal recovery mechanism. Just such a mechanism is naturally provided in the present nonisothermal extension of the complete potential framework discussed above. To illustrate the impact of the dynamic thermal recovery mechanism, the identical history as that described above was conducted (and is shown by a dashed line in Fig. 13) but with the removal of the $\theta_{i j} \dot{T}$ term in the internal constitutive rate equation, i.e., eq. (31). Although, for the present characterization and rate of thermal loading the influence was minimal, the inclusion of such a mechanism (as required by the potential structure) does provide an increase in the subsequent creep response. An entire study devoted to examining the importance of this new dynamic thermal recovery mechanism and its sensitivity to characterization and thermal loading rate will be the focus of future research.

\subsection{TMD Test}

As a final verification test a thermomechanical deformation (TMD) test was conducted, which involved a strain controlled (i.e., $\dot{e}=8.33 \times 10^{-4} / \mathrm{sec}$ ) tensile test at $300^{\circ} \mathrm{C}$ to $2.0 \%$ total strain, followed by an unload to zero load and then a reload under stress control (i.e., $\dot{\sigma}=68.95 \mathrm{MPa} / \mathrm{sec}$ ) to $103 \mathrm{MPa}$. Holding the load at $103 \mathrm{MPa}$, the temperature was then increased at a rate of $1^{\circ} \mathrm{C} / \mathrm{sec}$ to $650^{\circ} \mathrm{C}$; whereupon the load and temperature 


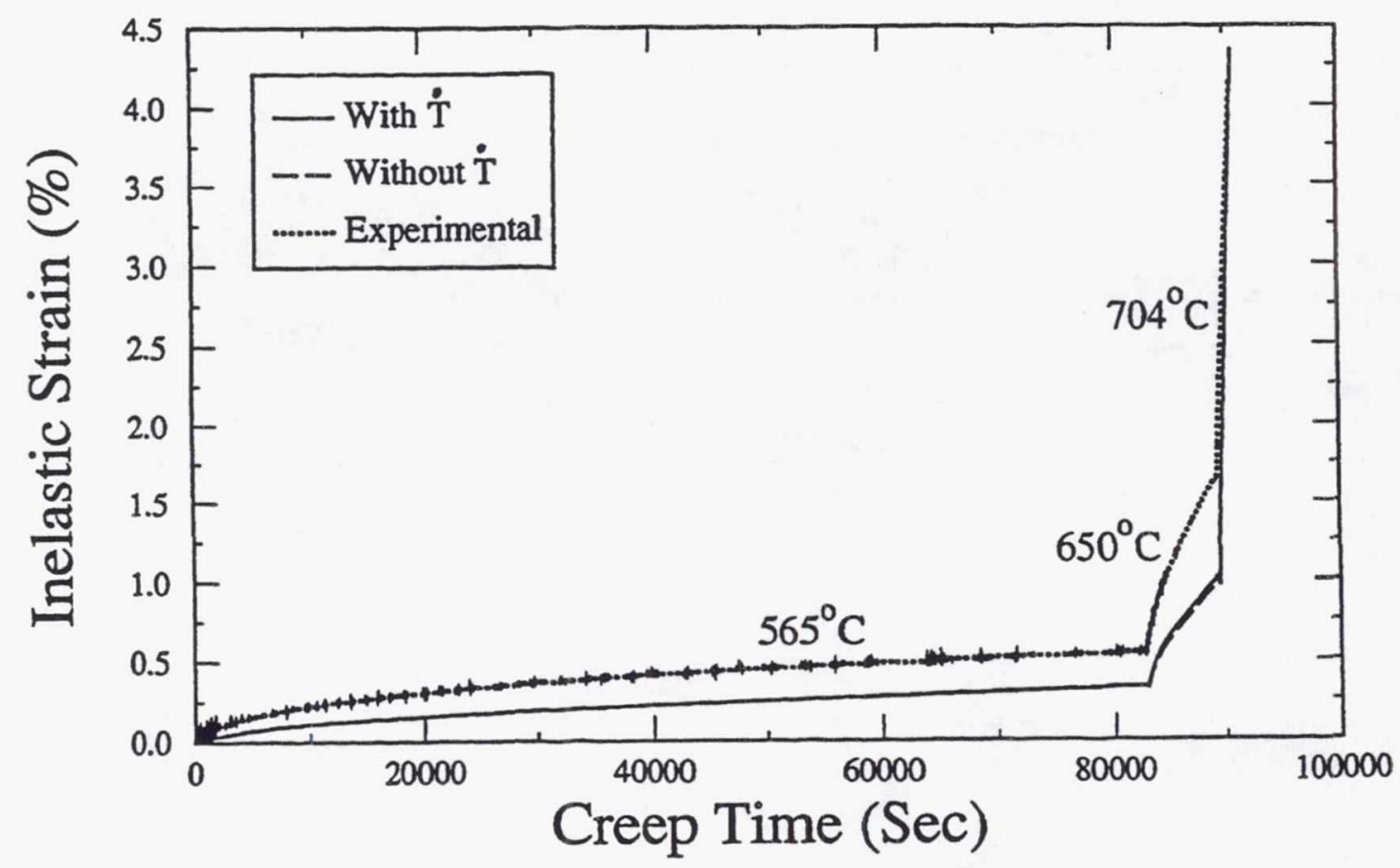

Figure 13: Prediction of multi-step temperature creep test, i.e., 565 to 650 to $704^{\circ} \mathrm{C}$, with and without the new dyanmic thermal recovery term.

were held fixed at their respective magnitudes for 8 hours. The resulting experimentally obtained stress-total strain and total strain versus time responses and model simulations are illustrated in Figs. 14, 15 and 16. Here, the thermal strain component was zeroed at the initial test temperature of $300^{\circ} \mathrm{C}$. Examining Fig. 14, it is obvious that the prior tensile overload is over-predicted, thus one might question the ability of the model to accurately predict the subsequent creep behavior. Part of the discrepancy in the initial tensile response can be attributed to the fact that the model exhibits a notable positive strain rate sensitivity at $300^{\circ} \mathrm{C}$ whereas the experimental data suggests a minimal strain rate dependence. Consequently, given that the characterization of the present model was performed at a strain rate of $8.333 \times 10^{-5} / \mathrm{sec}$ and the verification test was conducted at $8.33 \times 10^{-4} / \mathrm{sec}$, one would anticipate the present over-prediction of the tensile response. Further discrepancies can also be attributed to material variability ${ }^{6}$ and experimental scatter. Figures 15 and 16 show the comparison of the experimental and GVIPS simulation of the subsequent $103 \mathrm{MPa}$ TMD response. Again, the simulation under predicts the experimental results. Realizing that the experiment performed is actually a constant load test and not a constant stress creep test, as is the simulation, another simulation was conducted wherein the applied stress was increased by approximately $7 \%$ to account for the maximum change in cross sectional area of the test coupon. As a result, the

\footnotetext{
${ }^{6}$ The current TMD test coupon consisted of 12 plies, whereas all previous tensile test data were obtained from coupons constructed with 5 plies.
} 


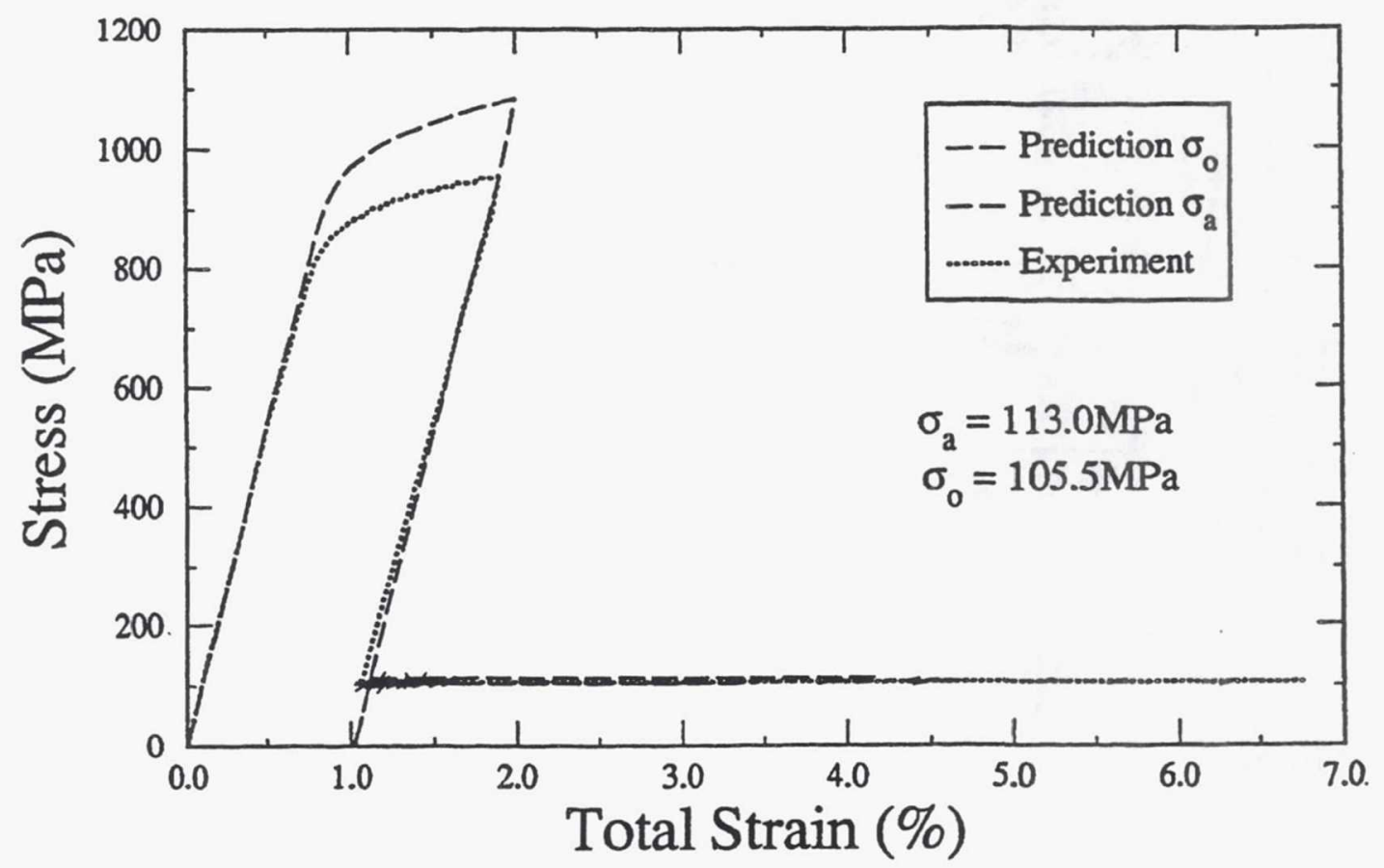

Figure 14: Prediction of stress-strain response for a thermomechanical deformation test.

accumulation of predicted inelastic strain was increased, thus providing an upper bound on the prediction, as shown by the dashed line in Fig. 16. Clearly, even with the inclusion of this additional increase in stress, the present characterization of the model still significantly under predicted the experimental results.

Examination of Fig. 15, which is an amplification of the transient temperature regime occurring within the first 600 seconds of the test provides some additional insight into the possible cause for the under-prediction of the present GVIPS model. For example, focusing upon the transient temperature region, one observes a nonlinear accumulation of strain starting at the 200 second mark and continuing on (but at a different rate) past the point at which the temperature becomes constant at $650^{\circ} \mathrm{C}$. This accumulation equals approximately $0.2 \%$ strain, which is precisely the amount of deviation between the simulation and the experimental observation at 600 seconds into the test; this suggests that the inelasticity predicted by the model at low stress levels and temperatures below $650^{\circ} \mathrm{C}$ is insufficient. This conclusion is further supported by the previous correlations shown in Fig. 4 where the model under-predicted the creep strain at 565 and $482^{\circ} \mathrm{C}$ by an increasing amount, as the temperature was decreased. Furthermore, the neglecting of the time dependent, reversible domain, as discussed previously may also contribute significantly to this nonlinear accumulation of strain during the temperature transient. Such areas will be the focus of future research. 


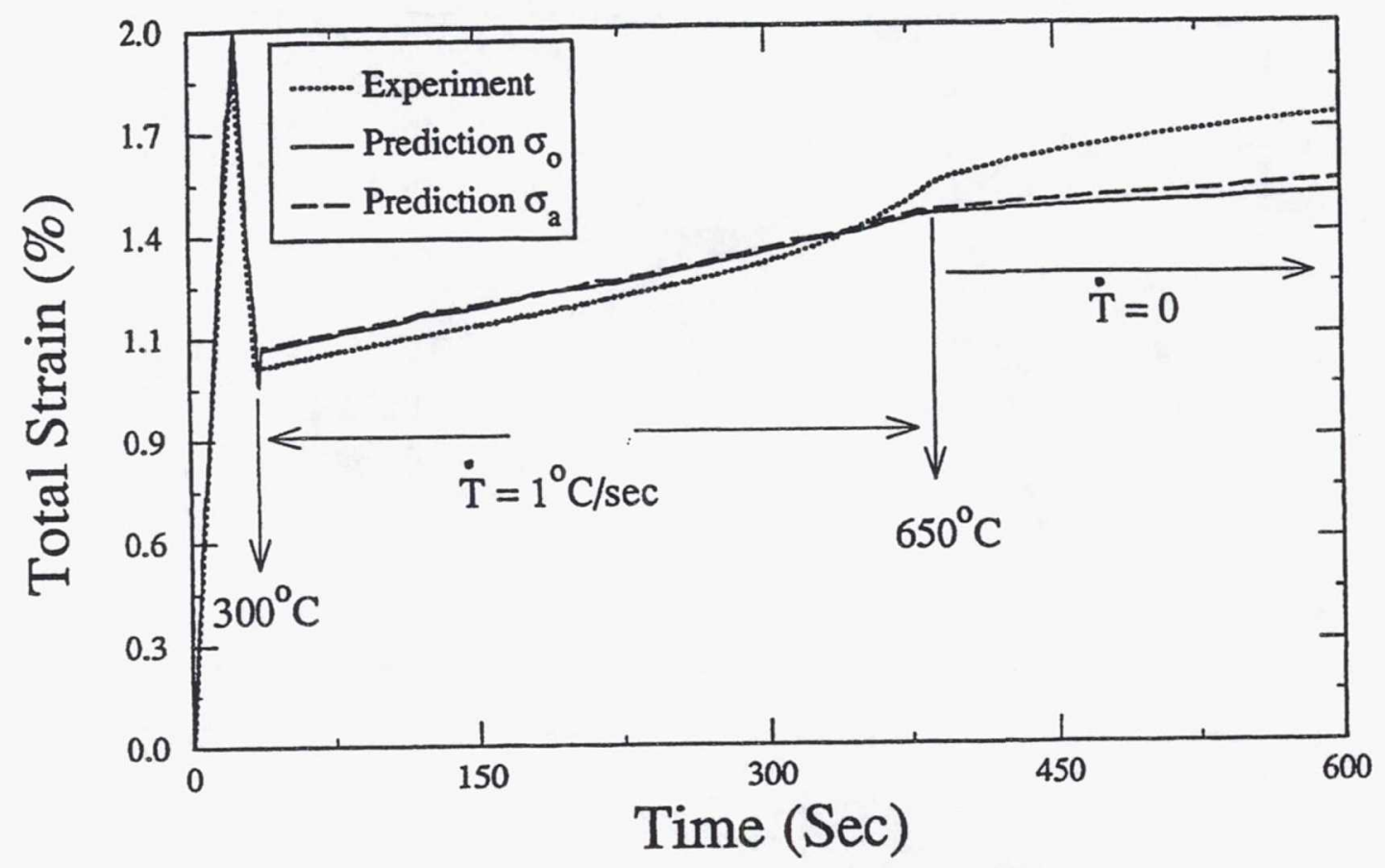

Figure 15: Prediction of short term total strain versus time response for a thermomechanical deformation test.

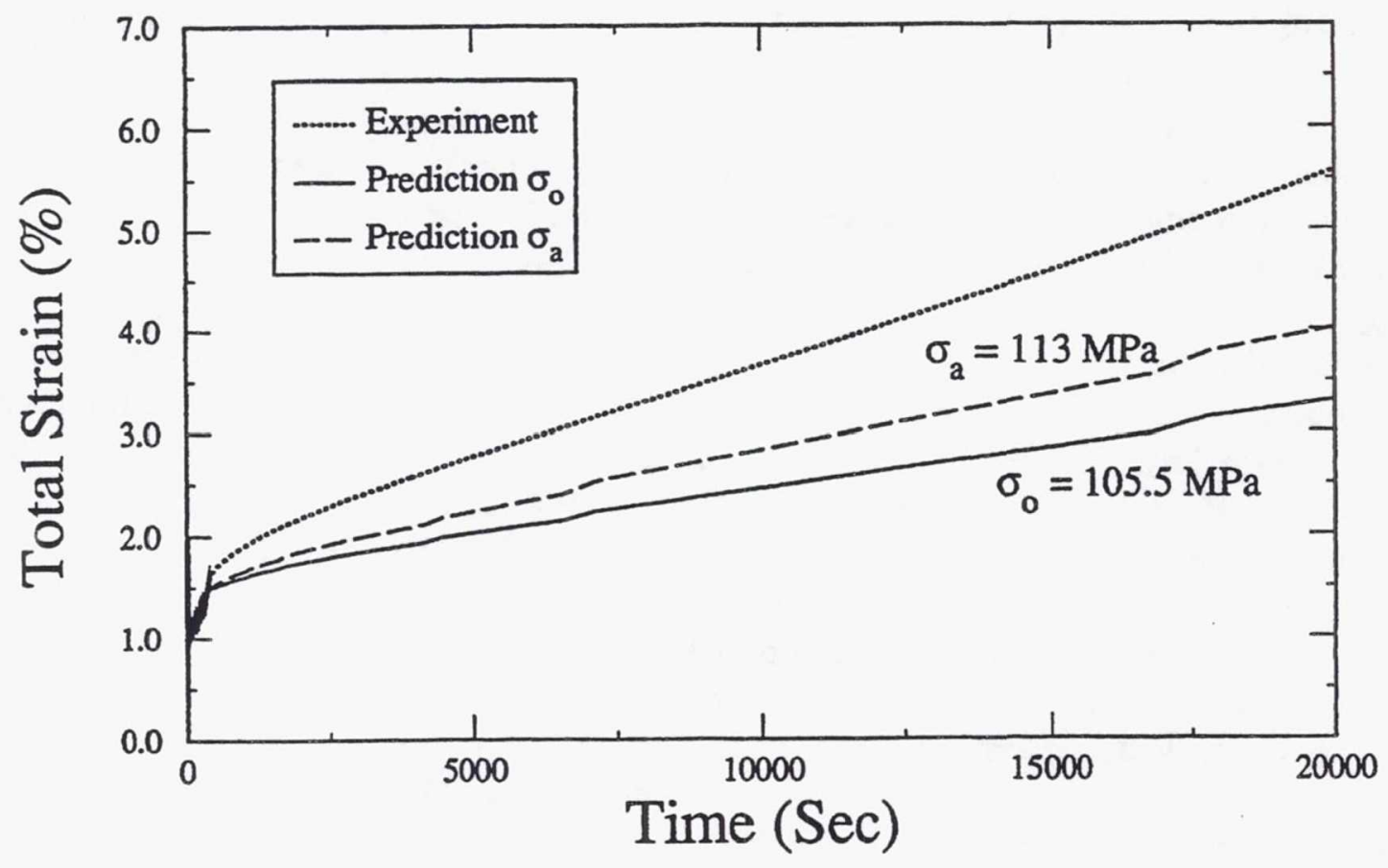

Figure 16: Prediction of long term total strain versus time response for a thermomechanical deformation test. 


\section{Summary}

A fully associative, multiaxial, nonisothermal, nonlinear kinematic hardening viscoplastic model has been presented. It contains three internal state variables (two scalars and one tensor) and both thermal and strain-induced recovery mechanisms. The two, nonevolving (yet temperature dependent), scalar internal state variables are associated with the dislocation density and are defined as the drag and yield stress. The evolving tensorial variable known as the internal (or back) stress is a second order, traceless, symmetric tensor and is associated with the dislocation substructure. A unique aspect of the present model is the inclusion of nonlinear hardening through the use of a compliance operator, derived from the Gibb's potential, in the evolution law for the back stress. This nonlinear tensorial operator is significant in that it allows both the flow and evolutionary laws to be fully associative (and therefore easily integrated), greatly influences the multiaxial response under non-proportional loading paths and in the case of nonisothermal histories, introduces an instantaneous thermal softening mechanism proportional to the rate of change in temperature. The resulting nonisothermal unified viscoplastic model was then characterized for the titanium based alloy TIMETAL 21S from room temperature to $704^{\circ} \mathrm{C}$. Results illustrated the good overall correlation and predictive capabilities of the model for a wide range of mechanical and thermal loading conditions.

\section{Acknowledgment}

Thanks be to God. We would also like to acknowledge the efforts of Dr. T.E. Wilt in the development of IDAC and implementation of this nonisothermal GVIPS model, and the reviewers for their helpful comments.

\section{References}

[1] Arnold, S.M.; and Saleeb, A.F.: On the Thermodynamic Framework of Generalized Coupled Thermoelastic Viscoplastic - Damage Modeling. NASA TM-105349, 1991.

[2] Saleeb, A.F. and Wilt, T.E.: Analysis of the Anisotropic Viscoplastic-Damage Response of Composite Laminates-Continuum Basis and Computational Algorithms, Int. Jnl. Num. Meth. Engng., Vol. 36, 1993, pp. 1629-1660.

[3] Arnold, S.M., Saleeb, A.F, and Wilt, T.E.: A Modeling Investigation of Thermal and Strain Induced Recovery and Nonlinear Hardening in Potential Based Viscoplasticity, NASA TM-106122, 1993.

[4] Saleeb,A.F., Seif, Y., and Arnold, S.M.: Fully-Associative Viscoplasticity with Anisotropic and Nonlinear Kinematic Hardening, submitted Int. Jnl. of Plasticity, 1994. 
[5] Arnold, S.M.; Saleeb, A.F., and Castelli, M.G.: A Fully Associative, Nonlinear Kinematic, Unified Viscoplastic Model for Titanium Based Matrices, NASA TM106609, 1994.

[6] Spencer, A.J.M.: Continuum Physics, Vol. 1, A.C. Eringen, Ed., Academic Press., London, 1971, p. 240.

[7] Lemaitre, J.; and Chaboche, J.L.: Mechanics of Solid Materials, Cambridge University. Press, New York, 1990.

[8] Orowan, E.: Causes and Effects of Internal Stresses, Internal Stresses and Fatigue of Metals, Proceedings of the Symposium on Internal Stresses and Fatigue in Metals, Detroit Mich., Eds., G.M. Rassweiler and W.L. Grvise, Elsevier Publishing, 1959.

[9] Robinson, D.N; and Swindeman, R.W.: Unified Creep-Plasticity Constitutive Equations for 2 1/4 Cr-1Mo Steel at Elevated Temperature, ORNL TM-8444, 1982.

[10] Miller, A.K., Ed.: Unified Constitutive Equations for Plastic Deformation and Creep of Engineering Alloys, Elsevier Applied Science, New York, 1987.

[11] Freed, A.D.; Chaboche, J.L.; and Walker, K.P.: A Viscoplastic Theory with Thermodynamic Considerations, Acta Mech, vol. 90, 1991, pp. 155-174.

[12] Neu, R.W.; Nonisothermal Material Parameters for the Bodner-Partom Model, MDVol. 43, Material Parameter Estimation for Modern Constitutive Equations, Eds, L.A. Bertram, S. B. Brown, and A. D. Freed, 1993, pp. 211-226.

[13] Sherwood, J. A. and Quimby, H.M.; "Micromechanical Modeling of Damage Growth in Titanium Based Metal-Matrix Composites", to appear Comp. Struc., 1995.

[14] Castelli, M.G., Arnold, S.M., and Saleeb, A.F.:Specialized Deformation Tests for the Characterization of a Viscoplastic Model: Application to a Titanium Alloy, NASA TM-106268, 1995.

[15] Ashbaugh, N.E., and Khobaib, M.: Unpublished Data, University of Dayton Research Institute, Dayton, Ohio. 


\section{APPENDIX A: Interpolation Functions}

Here, we list the functions and applicable temperature ranges used in this study to describe the five temperature dependent inelastic material parameters identified at discrete temperatures in Table 2. Note, although the magnitudes of the functions describing any one parameter are continuous across temperature boundaries, no attempt was made to ensure continuity of the slopes. This may be an extremely important fact depending upon the integration scheme utilized, and should be cautiously considered.

\begin{tabular}{|c|c|c|}
\hline \multirow{2}{*}{$\begin{array}{c}\text { Constants } \\
\kappa\end{array}$} & $\begin{array}{c}\text { Interpolation Functions } \\
a_{1}+b_{1}\end{array}$ & $\begin{array}{c}\text { Temperature Range, }{ }^{\circ} \mathrm{C} \\
T<565\end{array}$ \\
\hline & $\begin{array}{r}\left(1+\exp \left(-\frac{1-c_{1}}{d_{1}}\right)\right. \\
a_{2}+\frac{b_{2}}{T^{1.5}}+\frac{c_{2}}{T^{2}}\end{array}$ & $T \geq 565$ \\
\hline \multirow[t]{2}{*}{$\mu$} & $\frac{\mu_{0}}{\kappa_{0}} \kappa(T)\left[\exp \left(a_{3}+b_{3} T \ln (T)+c_{3} T^{2.5}\right)\right]$ & $T<565$ \\
\hline & $\frac{\mu_{0}}{\kappa_{0}} \kappa(T)\left[a_{4}+\frac{b_{4}}{\left(1+\exp \left(-\frac{\left(T-c_{4}\right)}{d_{4}}\right)\right.}\right]$ & $T \geq 565$ \\
\hline$B_{0}$ & $\begin{array}{c}a_{5}+b_{5} \exp \left(-\frac{T}{c_{5}}\right) \\
a_{6}+b_{6} T+c_{6} T^{2} \ln (T)+d_{d} T^{2.5}+e_{6} T^{3}\end{array}$ & $\begin{array}{l}T \leq 482 \\
T>482\end{array}$ \\
\hline \multirow{2}{*}{$R_{\alpha}$} & $4 \exp \left(\frac{-(T-c)}{d_{7}}\right)$ & $T-180$ \\
\hline & $\begin{array}{c}a_{8}-b_{8}(482-T)^{2} \\
0\end{array}$ & $\begin{array}{c}300 \leq T \leq 482 \\
T<300\end{array}$ \\
\hline$\beta$ & $\begin{array}{c}0.001 \\
0.001-1.0 \times 10^{-4} \\
0\end{array}$ & $\begin{array}{c}T \leq 100 \\
100<T \leq 200 \\
T>200\end{array}$ \\
\hline
\end{tabular}

where $\mu_{0}=5.52 \times 10^{-9}$ and $\kappa_{0}=5.86$. The above interpolation constants are:

$$
\begin{array}{llll}
a_{1}=-1027.4 & b_{1}=2118.5 & c_{1}=566.04057 & d_{1}=-154.9935 \\
a_{2}=254.76 & b_{2}=-1.997 \times 10^{7} & c_{2}=4.04 \times 10^{8} & \\
a_{3}=20.197653 & b_{3}=2.4519076 \times 10^{-3} & c_{3}=-3.41797 \times 10^{-6} & \\
a_{4}=-0.22876195 & b_{4}=3961.1386 & c_{4}=397.89176 & d_{4}=-31.582681 \\
a_{5}=5.782 \times 10^{-5} & b_{5}=1.003 \times 10^{-5} & c_{5}=-197.95617 & \\
a_{6}=-5.430 \times 10^{-2} & b_{6}=3.603 \times 10^{-4} & c_{6}=-4.006 \times 10^{-7} & d_{6}=1.156 \times 10^{-7} \\
a_{7}=1.670 \times 10^{-7} & b_{7}=1.0381 \times 10^{-4} & c_{7}=722.0 & e_{6}=-1.196 \times 10^{-9} \\
a_{8}=1.670 \times 10^{-7} & b_{8}=5.068 \times 10^{-12} & &
\end{array}
$$


Public reporting burden for this collection of information is estimated to average 1 hour per response, including the time for reviewing instructions, searching existing data sources, gathering and maintaining the data needed, and completing and reviewing the collection of information. Send comments regarding this burden estimate or any other aspect of this collection of information, including suggestions for reducing this burden, to Washington Headquarters Services, Directorate for Information Operations and Reports, 1215 Jefferso
Davis Highway, Suite 1204. Arlington, VA 22202-4302, and to the Office of Management and Budget, Paperwork Reduction Project (0704-0188), Washington, DC 20503.

1. AGENCY USE ONLY (Leave blank)

2. REPORT DATE

May 1995

3. REPORT TYPE AND DATES COVERED

Technical Memorandum

4. TITLE AND SUBTITLE

A Fully Associative, Nonisothermal, Nonlinear Kinematic, Unified Viscoplastic Model for Titanium Alloys

6. AUTHOR(S)

S.M. Arnold, A.F. Saleeb, and M.G. Castelli

WU-505-63-12

5. FUNDING NUMBERS

8. PERFORMING ORGANIZATION

REPORT NUMBER

National Aeronautics and Space Administration

Lewis Research Center

Cleveland, Ohio 44135-3191

E-9644

9. SPONSORING/MONITORING AGENCY NAME(S) AND ADDRESS(ES)

National Aeronautics and Space Administration

Washington, D.C. $20546-0001$

NASA TM-106926

11. SUPPLEMENTARY NOTES

Prepared for the Second Symposium on Thermomechanical Fatigue Behavior of Materials sponsored by the American Society for Testing and Materials, Phoenix, Arizona, November 13-18, 1994. S.M. Amold, NASA Lewis Research Center, A.F. Saleeb, University of Akron, Department of Civil

Engineering, Akron, Ohio 44325; and M.G. Castelli, NYMA, Inc., Engineering Services Division, 2001 Aerospace Parkway, Brook Park, Ohio 44142 (work funded by NASA Contract NAS3-27186). Responsible person, S.M. Amold, organization code 5220, (216) 433-3334.

12a. DISTRIBUTIONAVAILABILITY STATEMENT

12b. DISTRIBUTION CODE

Unclassified - Unlimited

Subject Category 39

This publication is available from the NASA Center for Aerospace Information, (301) 621-0390.

13. ABSTRACT (Maximum 200 words)

Specific forms for both the Gibb's and complementary dissipation potentials are chosen such that a complete (i.e., fully associative) potential based multiaxial, nonisothermal unified viscoplastic model is obtained. This model possess one tensorial internal state variable (that is, associated with dislocation substructure) and an evolutionary law that has nonlinear kinematic hardening and both thermal and strain induced recovery mechanisms. A unique aspect of the present model is the inclusion of nonlinear hardening through the use of a compliance operator, derived from the Gibb's potential, in the evolution law for the back stress. This nonlinear tensorial operator is significant in that it allows both the flow and evolutionary laws to be fully associative (and therefore easily integrated), greatly influences the multiaxial response under non-proportional loading paths, and in the case of nonisothermal histories, introduces an instantaneous thermal softening mechanism proportional to the rate of change in temperature. In addition to this nonlinear compliance operator, a new consistent, potential preserving, internal strain unloading criterion has been introduced to prevent abnormalities in the predicted stress-strain curves, which are present with nonlinear hardening formulations, during unloading and reversed loading of the external variables. The specific model proposed is characterized for a representative titanium alloy commonly used as the matrix material in SiC fiber reinforced composites, i.e., TIMETAL 21S. Verification of the proposed model is shown using "specialized" non-standard isothermal and thermomechanical deformation tests.

14. SUBJECT TERMS

Viscoplasticity, Nonlinear hardening; TIMETAL 21S; Nonisothermal; Deformation; Multiaxial; Correlations; Predictions

\begin{tabular}{|c|c|c|}
\hline $\begin{array}{c}\text { 17. SECURITY CLASSIFICATION } \\
\text { OF REPORT } \\
\text { Unclassified }\end{array}$ & $\begin{array}{c}\text { 18. SECURTY CLASSIFICATION } \\
\text { OF THIS PAGE } \\
\text { Unclassified }\end{array}$ & $\begin{array}{c}\text { 19. SECURITY CLASSIFICATION } \\
\text { OF ABSTRACT } \\
\text { Unclassified }\end{array}$ \\
\hline
\end{tabular}

NSN 7540-01-280-5500 